Supporting Information

\title{
Analysis of Diffusivity Change from Single-molecule Trajectories on Living Cells
}

Rong Zhao, ${ }^{+,+,}, J^{\perp}$ inghe Yuan, ${ }^{*+,}, \perp$ Nan Li, ${ }^{+,+}$Yahong Sun, ${ }^{+, \$}$ Tie Xia," and Xiaohong Fang ${ }^{*,+, \neq}$

${ }^{+}$Beijing National Research Center for Molecular Sciences, Key Laboratory of Molecular Nanostructure and Nanotechnology, Institute of Chemistry, Chinese Academy of Sciences, Beijing 100190, P. R. China

${ }^{\ddagger}$ University of Chinese Academy of Sciences, Beijing 100049, P. R. China

${ }^{\S}$ The Second High School Attached to Beijing Normal University, Beijing 100088, P. R. China

"Institute for Immunology, School of Medicine, Tsinghua University, Beijing 100084, China

\section{Contents}

- Proof of Rayleigh distribution

- Proof of 2D restricted motion obeying Rayleigh distribution approximatively

- Synthesis of diffusion displacement data

- Definition of various errors

- Validation on synthetic data

- Application to experimental data

- Experimental procedures

- $\quad$ Supporting Figures and Tables

- Supporting References 


\section{Proof of Rayleigh distribution}

From the probability density function (PDF) of diffusion displacements in the two orthotropic dimensions

$$
\left\{\begin{array}{l}
p(x)=\frac{1}{\sqrt{2 \pi} \sigma} \exp \left(\frac{-x^{2}}{2 \sigma^{2}}\right) \\
p(y)=\frac{1}{\sqrt{2 \pi} \sigma} \exp \left(\frac{-y^{2}}{2 \sigma^{2}}\right)
\end{array}\right.
$$

we know that the joint probability density function of $(x, y)$ is

$$
p(x, y)=p(x) p(y)=\frac{1}{2 \pi \sigma^{2}} \exp \left(-\frac{x^{2}+y^{2}}{2 \sigma^{2}}\right)
$$

Hence, the cumulative distribution function of $z=\sqrt{x^{2}+y^{2}}$ can be derived

$$
F(z)=\iint_{A} p(x, y) d x d y=\frac{1}{2 \pi \sigma^{2}} \iint_{A} \exp \left(-\frac{x^{2}+y^{2}}{2 \sigma^{2}}\right) d x d y
$$

where the integral area $A$ is the interior of a circle with radius $z$.

$$
F(z)=\frac{1}{2 \pi \sigma^{2}} \int_{0}^{2 \pi} d \varphi \int_{0}^{Z} \exp \left(-\frac{r^{2}}{2 \sigma^{2}}\right) r d r=\frac{1}{\sigma^{2}} \int_{0}^{Z} r \exp \left(-\frac{r^{2}}{2 \sigma^{2}}\right) d r
$$

The PDF of $r$ is its derivative respect to $z$

$$
p(r)=\frac{r}{\sigma^{2}} \exp \left(-\frac{r^{2}}{2 \sigma^{2}}\right),(r>0)
$$

\section{Proof of 2D restricted motion obeying Rayleigh distribution approximatively}

We start with the one-dimensional restricted motion. Consider one-dimensional diffusion is confined between two reflecting boundaries at $\mathrm{x}=0$ and $L$. Let $P\left(x_{2}, t \mid x_{1}, 0\right)$ be the probability that the position of a particle is $x_{2}$ at time $\mathrm{t}$, given that it is $x_{1}$ at $\mathrm{t}=0$. According to the reference ${ }^{1}$, the probability distribution of one-dimensional restricted motion can be described as

$$
\begin{gathered}
P\left(x_{2}, t \mid x_{1}, 0\right)=\frac{1}{\mathrm{~L}}+\frac{2}{\mathrm{~L}} \sum_{n=1}^{\infty} \cos \left(\frac{n \pi x_{1}}{L}\right) \cos \left(\frac{n \pi x_{2}}{L}\right) \exp \left\{-\frac{1}{2}\left(\frac{n \pi \sigma}{L}\right)^{2} t\right\} \quad(t>0) \\
P\left(x_{2}, t \mid x_{1}, 0\right)=\delta\left(x_{2}-x_{1}\right) \quad(t=0) \quad(\mathrm{ES} 7)
\end{gathered}
$$

Based on this formula, let $\mathrm{t}$ equals to the exposure time of one frame, the probability distribution can be represented in Figure S1a. When $x_{1}=L / 2$, the probability distribution (black line in Figure S1b) is exactly the same as the probability distribution of Brownian diffusion with the same diffusion coefficient (red dotted line in Figure S1b).

As proven in Methods of main text, the diffusion displacement of Brownian diffusion along 
each dimension is modeled as normal distribution, and when expending to $2 \mathrm{D}$, the probability distribution of the diffusion displacement is a Rayleigh distribution. Therefore, when the initial position of particles is at the center of the restricted area, 2D restricted motion can be described by Rayleigh distribution completely. When it deviates from the center $(L / 2)$ of the restricted area less than $L / 3$, the probability distribution (black line in Figure S1c) can also be fitted approximately by the probability distribution of Brownian diffusion with the same diffusion coefficient ${ }^{2}$ (red dotted line in Figure S1c). In summary, 2D restricted motion can be described by Rayleigh distribution for particles in most restricted areas where their positions are not too close to the boundaries.

\section{Synthesis of diffusion displacement data}

Although a discretized virtual cell membrane geometry can be used to synthetize the diffusion displacement data, ${ }^{3}$ the discretized step size will affect the diffusion displacement distribution evidently and it is very time-consuming for the synthesis of slow-diffusion data. For free diffusion and restricted diffusion, it is proven that the diffusion displacement in one-dimension defer to normal distribution. Therefore, we synthesized the diffusion displacement data as following.

Given the state number $K$, the state transition probability matrix $A$ and the initial state probability distribution $\pi$, we generated $(N \times L)$ random $K$-state Markov sequences with single sequence length $L$ and sample number $N$. Then refer to the states in the Markov sequences, we generated $(N \times L)$ one-dimensional displacement data $x$ and $y$ of normal distribution $N\left(0, \quad \sigma_{i}^{2}=2 D_{i} \tau+\left\langle(\Delta x)^{2}\right\rangle\right)$ respectively. It can be proven that the diffusion displacement $\left(r=\sqrt{x^{2}+y^{2}}\right)$ is in Rayleigh mixture distribution (Equation 4).

\section{Definition of various errors}

In all the experiments on synthetic data, we estimated the variances from eleven calculations. The state number bias is defined as the deviation between the estimated state number and the actual state number. The variance of the state number is the overall variance of estimated state number from all eleven calculations. 
The state dwell time bias is defined as the ratio of the counts of wrong state to the whole actual state in the state path. This bias would influence the estimates of the state mixture ratios and transition probability.

The diffusion coefficient bias is defined as the ratio of the difference between the estimated diffusion coefficient and actual diffusion coefficient to the actual diffusion coefficient.

The transition probability bias is defined as the difference between the estimated transition probability and the actual transition probability.

The state mixture ratio bias is defined as the difference between the estimated state mixture ratio and the actual state mixture ratio.

\section{Validation on synthetic data}

\section{On diffusion coefficient separation}

To check the influence of diffusion coefficient separation on the recovery accuracy, refer to the experimental diffusion coefficients of membrane proteins, we synthesized the diffusion displacement data with 2-state diffusion (Table S4). The procedure to generate the synthetic data is described above.

Given the state transition probability matrix $A$ and the initial state probability distribution $\pi$, we generated $(N \times L)$ random 2-state Markov sequences with sample number $N=3000$ and sequence length $L=50$ frames for each sample. Then refer to the states in the Markov sequences, under the localization uncertainty $\sqrt{\left\langle(\Delta x)^{2}\right\rangle}=10,20,30,40$ and 50nm, we generated $(N \times L)$ displacement data $x$ and $y$ of normal distribution $N\left(0, \quad \sigma_{i}^{2}=2 D_{i} \tau+\left\langle(\Delta x)^{2}\right\rangle\right)$ respectively. It can be proven that the diffusion displacement $\left(r=\sqrt{x^{2}+y^{2}}\right)$ is in Rayleigh mixture distribution (equation 4 with $K=2$ in the Methods).

With MLE method as mentioned in the Methods of the main text, we estimated the variance $\sigma_{i}^{2}$ and hence $D_{i}$ for fixed state number $K$. By setting maximum probable state number $K_{\max }=10$, using the histogram of the diffusion displacement data to approximate the PDF of the diffusion displacement, and calculating its deviation from the estimated Rayleigh mixture distribution under 
increasing state number $K$, we can determinate the most probable state number $K_{p}$ with the least deviation, namely, $K_{p}=2$.

We trained the HMM by initializing the state number with $K_{p}=2$, the state transition probability matrix randomly, the initial state probability distribution with $\widehat{\alpha}$, and fixing the emission probability as $p\left(r \mid \omega_{i}, \hat{\sigma}_{i}^{2}\right)$, and then put this trained HMM to the synthetic diffusion displacement data to extract the initial state probability distribution $\hat{\pi}$, the state transition probability matrix $\hat{A}$. With the Viterbi algorithm, we extracted the most probable state path of every trajectory and further calculated the state mixture ratios and state dwell time of every state.

Figures S2 and S3 show the biases and standard deviations of the four parameters under the localization uncertainty $10-50 \mathrm{~nm}$ and different diffusion coefficients separation $0.9231 \leq$ $\left(D_{2}-D_{1}\right) / D_{1} \leq 18.23$. Under all the localization uncertainty and diffusion coefficients separation, MLE estimated the state number correctly (Figure S2a). Under all the localization uncertainty, with the increase of diffusion coefficients separation, the diffusion coefficient biases (Figure S2c) eventually fluctuate at about $3 \%$ for $D_{1}$ (left), only $1 \%$ for $D_{2}$ (right). Even in the worst situation, the diffusion coefficient biases are acceptable $\left(14.0 \%\right.$ for $D_{1}$ and $4.2 \%$ for $\left.D_{2}\right)$. Under the localization uncertainty $10 \mathrm{~nm}$ and $20 \mathrm{~nm}$, when $\left(D_{2}-D_{1}\right) / D_{1} \geq 1.69$, the transition probability biases are less than 0.03 (Figure S3). But under the localization uncertainty larger than $30 \mathrm{~nm}$ (Figure S3), to get the same transition probability biases, larger diffusion coefficients separation is needed. We can always get acceptable state dwell time biases under all the localization uncertainty (Figure S2b). Even in the worst situation, the state dwell time bias is $27.1 \%\left(\left(D_{2}-D_{1}\right) / D_{1}=0.92\right.$ and localization uncertainty 50nm).

\section{On varied trajectory number}

To check the dependence of recovery accuracy on the trajectory number, under the localization uncertainty $10 \mathrm{~nm}, 20 \mathrm{~nm}, 30 \mathrm{~nm}$ and $40 \mathrm{~nm}$, we synthesized a series of 3-state diffusion displacement data with a fixed trajectory length ( $L=20$ frames) and a varied trajectory number $N=100-10000$ (Table S5). The procedure to generate the synthetic data and to estimate the parameters with MLE/HMM are similar to that in the previous section. 
Under the localization uncertainty $\sqrt{\left\langle(\Delta x)^{2}\right\rangle}=10 \mathrm{~nm}, 20 \mathrm{~nm}, 30 \mathrm{~nm}$ and $40 \mathrm{~nm}$, the MLE estimated the state number correctly when the trajectory number $N \geq 600,700,1000$ and 2700 respectively (Figure S4a).

Under the localization uncertainty $10 \mathrm{~nm}$ and $20 \mathrm{~nm}$, with the increase of trajectory number, the diffusion coefficient biases eventually fluctuate at about $9 \%$ for $D_{1}$ (left), $10 \%$ for $D_{2}$ (middle) and only $2 \%$ for $D_{3}$ (right). It is still acceptable although there are larger biases and standard deviations in the diffusion coefficient under the localization uncertainty $30 \mathrm{~nm}$ and $40 \mathrm{~nm}$ (Figure $\mathrm{S} 4 \mathrm{c})$.

Under the localization uncertainty $10 \mathrm{~nm}$ and $20 \mathrm{~nm}$, the biases of the transition probability between different states are nearly zeros, while standard deviations under the localization uncertainty $20 \mathrm{~nm}$ are larger (Figure S5). Under the localization uncertainty $30 \mathrm{~nm}$ and $40 \mathrm{~nm}$, the biases of transition probability become larger, especially for the transition probability $A_{22}$. Even if the trajectory length further increases, we cannot get smaller biases (Figure S5).

With the increasing of localization uncertainty, the biases and standard deviations of the state dwell time increased correspondingly (Figure S4b).

\section{On varied single trajectory length}

Under the localization uncertainty $10 \mathrm{~nm}, 20 \mathrm{~nm}, 30 \mathrm{~nm}$ and $40 \mathrm{~nm}$, we synthesized the diffusion displacement data with 3-state diffusion to check the recovery accuracy at different trajectory length as well (Table S6). The single trajectory length $L$ varied from 10 to 500 frames and trajectory number is fixed at $N=1000$.

Under the localization uncertainty $10 \mathrm{~nm}$ and $20 \mathrm{~nm}$, MLE estimated all the state number correctly, and there are a few wrong estimated state numbers under the localization uncertainty $30 \mathrm{~nm}$, but when the localization uncertainty is larger than $40 \mathrm{~nm}$, MLE can hardly estimate the state number (Figure S6a).

From Figure S6c, we can see that, with the increased localization uncertainty, larger biases and standard deviations appear in the diffusion coefficients. But under the localization uncertainty 10 $\mathrm{nm}$ and $20 \mathrm{~nm}$, the biases and standard deviations in the diffusion coefficients are roughly equal, and the diffusion coefficient biases fluctuate at about $12 \%$ for $D_{1}$ (left), $25 \%$ for $D_{2}$ (middle) and only $2 \%$ for $D_{3}$ (right). 


\section{On varied state number}

To check the influence of state number on the recovery accuracy of state dwell time, we synthesized a series of diffusion displacement data with 2 to 5 states and kept both dwell time of each state and the diffusion coefficient separation roughly the same. The single trajectory length $L$ varied from 200 to 500 frames and trajectory number was fixed at $N=2000$. The procedure to generate the synthetic data and to estimate the parameters with MLE/HMM were similar to that in the previous section.

As shown in Figure S8, with the increase of state number in a trajectory, the state dwell time bias increase almost linearly. When the state number increases to 5 , the state dwell time bias fluctuate at about $32 \%$, which is still acceptable.

\section{On varied state mixture ratio}

To check the influence of state mixture ratio on the recovery accuracy of state number. We synthesized a series of diffusion displacement data with 2 states. The single trajectory length and trajectory number are fixed at $\mathrm{L}=50$ and $\mathrm{N}=2000$. The diffusion coefficients of state 1 and state 2 are $0.0260 \mu \mathrm{m}^{2} / \mathrm{s}$ and $0.0790 \mu \mathrm{m}^{2} / \mathrm{s}$ respectively, which are similar to the diffusion of dimeric and monomeric receptors on cell membrane (Table S7).

As shown in Figure S9, when the mixture ratio of a state is larger than 5\%, the state number can be estimated completely correct. When the mixture ratio of a state is between $1 \%$ and $5 \%$, it is possible to generate erroneous estimates during eleven calculations, the error rate is $45 \%$ for the slow diffusion state 1 at the mixture ratio of $2 \%$, and $64 \%$ for fast diffusion state 2 at the mixture ratio of $1 \%$. When the mixture ratio of a state is less than $1 \%$, the state number can hardly be estimated correctly. Overall, for the state with its ratio above $5 \%$, our method can accurately identify the state number.

\section{Comparison of the performance of RmHMM and vbSPT}

We compared the performance of RmHMM with the vbSPT method using simulated trajectories and checked the effects of trajectory number on the recovery accuracy of both methods. We synthesized a series of 3-state diffusion trajectories with a mean trajectory length of around 20 frames $(2<\mathrm{L}<40)$ and a varied trajectory number $(\mathrm{N}=100-10000)$ in which the diffusion coefficients were similar to those of membrane proteins (Table S8). 
To imitate the real experimental data analysis in practice, where the real number of diffusion states is not prior knowledge, the maximum of hidden states $\left(K_{\max }\right)$ cannot be set too small. We set it to 8 in RmHMM and 10 in vbSPT according to their recommended values. However, even when the trajectory number $N=10000$, the vbSPT method falsely estimated that the state number $(K)$ as 6 . Then we reduced the $K_{\max }$ from 10 to 6 and found the $K$ was always falsely estimated as 6 . When the $K_{\max }$ was set to 5,4 or 3 separately, the $K$ was estimated as 5, 4 or 3 correspondingly. In contrast, using RmHMM, the state number can always be estimated as 3 correctly. It demonstrated that vbSPT was unsuitable for analyzing diffusivity change of membrane proteins, which was explained in the introduction part of main text that some underlying assumption in vbSPT would often be false in two-dimensional systems. Overall, for intracellular molecules that diffuse rapidly $\left(D>0.1 \mu \mathrm{m}^{2} / \mathrm{s}\right)$, it has been confirmed that vbSPT can estimate changes of diffusion states accurately. But for membrane proteins, which diffuse slower $\left(D<0.1 \mu \mathrm{m}^{2} / \mathrm{s}\right)$, it has been revealed that vbSPT tends to overfit and find excessive states.

In addition, when we pre-determined the state number as $3\left(K_{\max }=3\right)$ in vbSPT, we compared the recovery accuracy of two methods on diffusion coefficient, state mixture ratio and state transition probability (Figure S10). It showed that the diffusion coefficients estimated by RmHMM were much more accurate than them of vbSPT. And for state mixture ratio and state transition probability, RmHMM can get accurate results with fewer trajectories.

Therefore, our RmHMM method performs better than the other method, especially for the slowdiffusion membrane proteins.

\section{Application to experimental data}

\section{On EGFR data}

Refer to the formula of the localization uncertainty in the two lateral dimensions, ${ }^{4}$

$$
\sqrt{\left\langle(\Delta x)^{2}\right\rangle}=\sqrt{\left\langle(\Delta y)^{2}\right\rangle}=\sqrt{\frac{s^{2}+a^{2} / 12}{N}+\frac{4 \sqrt{\pi} s^{3} b^{2}}{a N^{2}}}
$$

where the standard deviation of the point spread function $s$, number of the collected photons $N$, and the background noise $b$ can be calculated with Gaussian fitting the single spark in the images, and $a$ is the pixel size of the sensor. We calculated the localization uncertainty of EGFR 
trajectories. Figure S11a shows the comparison between diffusion displacement (green stars) and localization uncertainty (red circles), and Figure S11b shows the localization uncertainty distribution (black bars) in two orthotropic $(\mathrm{X} / \mathrm{Y})$ dimensions and the fitted Gaussian mixture models (red dashed curves) with the expected value $\mu_{i}$ and the mixture ratio $\alpha_{i}$ (the inset tables show the parameters of the Gaussian mixture models).

From the fitted Gaussian mixture models, we obtained the mean localization uncertainty in the two dimensions is all $22.3 \mathrm{~nm}$ (corresponding to diffusion coefficients of $0.0025 \mu \mathrm{m}^{2} / \mathrm{s}$ at the frame rate $10 \mathrm{~Hz}$ ), thus we used $22.3 \mathrm{~nm}$ localization uncertainty in the calculation.

Mixing up the trajectory data of diffusion displacements from single-molecule imaging of EGFR in resting and stimulated HeLa cells, with the MLE algorithm, we got the estimated most probable state number $K_{p}$, diffusion coefficients $\widehat{D}$, and the Rayleigh mixture ratios $\widehat{\alpha}$. Figure S12a shows the MLE errors with the increase of state number, where MLE error is defined as the deviation value between the PDF of diffusion displacements and the estimated Rayleigh mixture distribution. The most probable state number $K_{p}$ is determined at the least MLE error, namely, $K=5$ in Figure S12a. It should be noticed that, because the determinate Rayleigh probability distribution, more state number does not guarantee smaller MLE error.

Bootstrapping was used in the MLE to get robust estimates, in which, the total bootstrap number is 11 and $90 \%$ stochastic data of the data set was used in each iteration, the estimated most probable state number is selected as the state number. For example, in Figure S12b, there are 7 times when the state number is estimated as 4 in 11 calculations. $K_{p}=4$ is selected as the state number.

Figure S13 shows the PDF (normalized histogram) of the diffusion displacements of EGFR trajectories in black solid curve and the estimated 4-state Rayleigh mixture distribution in red dashed curves, and the inset table are the estimated parameters with MLE.

By initializing the state number with $K_{p}=4$, the state transition probability randomly, the initial state probability distribution with $\hat{\alpha}$, and fixing the emission probability as the 4 Rayleigh distributions $p\left(r \mid \omega_{i}, \hat{\sigma}_{i}^{2}\right)$, we trained the HMM with the trajectory data mentioned above (Table S9).

Finally, we put this trained HMM to the EGFR trajectory data from the resting and the stimulated HeLa cells separately, to extract their initial state probability distribution $\hat{\pi}$ and the transition probability $\hat{A}$ respectively, and meanwhile to get the most probable state path of every trajectory 
with Viterbi algorithm and further to calculate the state dwell time and state mixture ratio of every state.

Table S1 shows the extracted parameters in four diffusion states and in which, the rows labeled with "Resting" and "Stimulated" stand for the resting HeLa cells and EGF stimulated HeLa cells respectively. Figure S14a is a representative trajectory with unusual transition between the state 3 and state 4, and Figure S14b shows the exponential decay of the dwell time of the $4^{\text {th }}$ state with characteristic time $\tau_{4}=0.09 \mathrm{~s}$ (smaller than the exposure time $0.1 \mathrm{~s}$ ). Hence we did not analyze the $4^{\text {th }}$ states in detail, and hypothetically attributed them to the large localization uncertainty or miscollecting.

To validate the registration of the slow $\left(2^{\text {nd }}\right)$ and fast $\left(3^{\text {rd }}\right)$ diffusion states to dimeric EGFR and monomeric EGFR states, an EGFR mutant DelD (deletion of the entire dimerization arm) (Figure S15) was examined. We applied our RmHMM method to analyze DelD trajectories from both resting and EGF-treated Hela cells (Table S10). The RmHMM method also extracted four diffusion states from all the trajectories (Table S2).

As shown from the RmHMM analyses, the DelD also exhibited two major diffusion states with diffusion coefficients $D_{2}=0.0213 \pm 0.0025 \mu \mathrm{m}^{2} / \mathrm{s}$ and $D_{3}=0.0691 \pm 0.0050 \mu \mathrm{m}^{2} / \mathrm{s}$, which were considered as dimer and monomer, respectively.

\section{On TßRII data}

The method was performed on T $\beta$ RII-GFP trajectories ( $L \geq 20$ frames for each trajectory) from cells with or without TGF- $\beta$ stimulation (Table S11).

Mixing up the trajectory data of diffusion displacements from single-molecule imaging of T $\beta R I I$ in resting and stimulated HeLa cells, with the MLE algorithm, we got the estimated most probable state number $K_{p}=5$, diffusion coefficients $\widehat{D}$, and the Rayleigh mixture ratios $\hat{\alpha}$.

Figure S16 shows the PDF (normalized histogram) of the diffusion displacements in black solid curve and the estimated 5-state Rayleigh mixture distribution in red dashed curves, and the inset table are the estimated parameters with MLE.

By initializing the state number with $K=5$, the state transition probability randomly, the initial state probability distribution with $\hat{\alpha}$, and fixing the emission probability as the 5 Rayleigh distributions $p\left(r \mid \omega_{i}, \hat{\sigma}_{i}^{2}\right)$, we trained the HMM with the trajectory data as mentioned above. (Table 
Finally, we put this trained HMM to the T $\beta$ RII trajectory data from the resting and the stimulated HeLa cells separately, to extract their initial state probability distribution $\hat{\pi}$ and the transition probability distribution matrix $\hat{A}$ respectively, and meanwhile to get the most probable state path of every trajectory with Viterbi algorithm and further to calculate the state dwell time and state mixture ratio of every state. Table S12 shows the extracted parameters in five diffusion states and in which, the rows labeled with "Resting" and "Stimulated" stand for the resting HeLa cells and TGF- $\beta$ stimulated HeLa cells respectively.

\section{Experimental Procedures}

\section{Cell culture and transfection}

Plasmids of EGFR-EGFP and TRRII-EGFP were constructed as previously described. ${ }^{5}$ The EGFR mutant DelD was generated by deletion the residues $242-259$ of EGFR-EGFP. ${ }^{6}$ All the plasmids were confirmed by DNA sequencing.

HeLa cells were cultured in Dulbecco's modified Eagle's medium (DMEM, Gibco) supplemented with $10 \%$ fetal bovine serum (Hyclone) and antibiotics ( $50 \mathrm{mg} / \mathrm{mL}$ streptomycin, 50 $\mathrm{U} / \mathrm{mL}$ penicillin) at $37{ }^{\circ} \mathrm{C}$ in a $5 \% \mathrm{CO}_{2}$ atmosphere. Cells were seeded in a $35-\mathrm{mm}$ glass-bottom dish for 16 hours and then transfected with $0.5 \mu \mathrm{g}$ T $\beta$ RII-EGFP, EGFR-EGFP or DelD-EGFP plasmids in the DMEM medium for 4 hours, respectively. Transfection was performed using Lipofectamine 2000 (Invitrogen) according to manufacturer's instructions.

For the ligand stimulation experiments, cells transfected with EGFR-EGFP or DelD-EGFP were added with $10 \mathrm{ng} / \mathrm{ml} \mathrm{EGF} \mathrm{(R \& D)} \mathrm{in} \mathrm{phenol} \mathrm{red-free} \mathrm{DMEM} \mathrm{for} 10 \mathrm{~min}$ at $37^{\circ} \mathrm{C}$ before fluorescence imaging. Cells transfected with T $\beta$ RII-EGFP were added with $10 \mathrm{ng} / \mathrm{ml}$ TGF- $\beta$ (R\&D) in phenol red-free DMEM for $15 \mathrm{~min}$ at $37^{\circ} \mathrm{C}$ before fluorescence imaging.

\section{Single-molecule imaging}

Single molecule fluorescence imaging was performed by a home-built TIRF microscope using an inverted Olympus IX71 microscope equipped with a total internal reflective fluorescence 
illuminator, a 100×/1.45 NA Plan Apochromatic TIRF objective and an electron-multiplying chargecoupled device (EMCCD) camera (Andor iXon DU-897D BV). EGFP molecules were excited by a $488 \mathrm{~nm}$ laser at $1 \mathrm{~mW}\left(60 \mathrm{~W} / \mathrm{cm}^{2}\right)$ (Melles Griot, Carlsbad, CA, USA). The collected fluorescent signals were passed through a filter HQ 525/50 (Chroma Technology), and then directed to the EMCCD camera. The gain of the EMCCD camera was set at 300. Movies of 300 frames were acquired for each sample at a frame rate of $10 \mathrm{~Hz}$.

\section{Single molecule tracking with U-Track software}

Time-lapse sequences of single-molecule image were acquired and then tracked with U-Track methods as described in reference. ${ }^{7}$ By fitting Gaussian kernels to approximate the two-dimensional point spread function of the microscope objective around local intensity maxima, the sub-pixel localization is achieved. To construct the trajectories, the algorithm first links the detected particles between consecutive frames, and then links the generated track segments to simultaneously close gaps and capture particle merging and splitting events.

\section{Statistics analysis}

For robust single-molecule analysis, we repeated the experiment three times to obtain three sets of data under each condition, and each set of data included thousands of single-molecule trajectories from 6 to 8 cells to be analyzed by RmHMM.

The diffusion coefficient, mixture ratio and transition probability were shown as mean \pm the standard deviation from three experiments. The results obtained from cells with ligand stimulations were compared to those of the resting cells using the unpaired Student's t-test. (GraphPad Prism Software). The state dwell times were obtained from fitting of histograms of thousands trajectory segments and they were compared by non-parametric Mann-Whiteney U test. P-value less than 0.05 is regarded as statistically significant. 
a

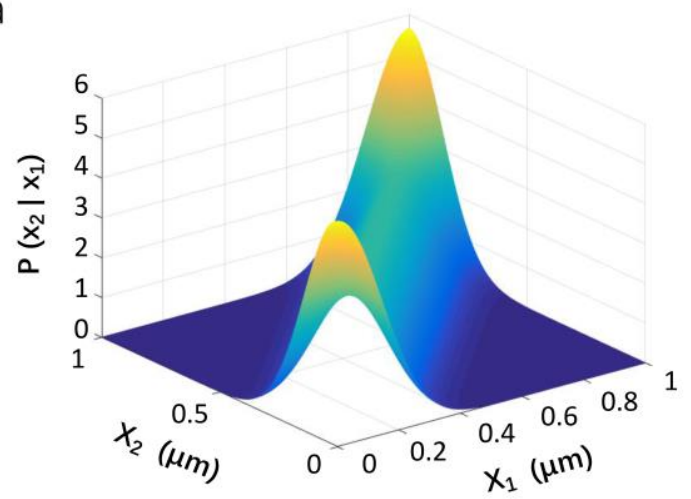

$b$

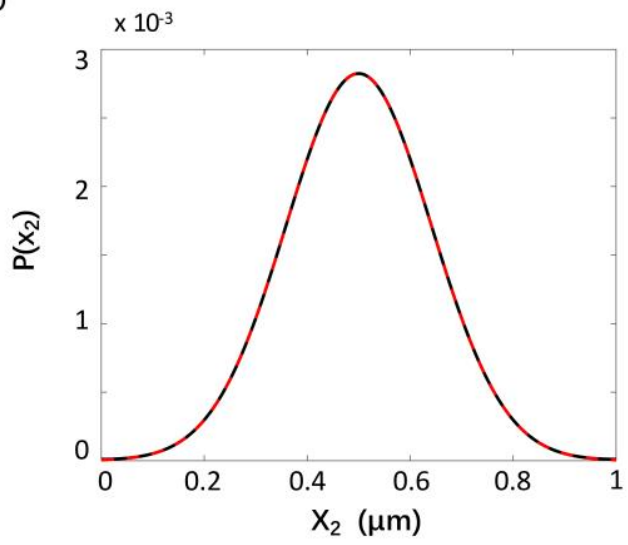

C

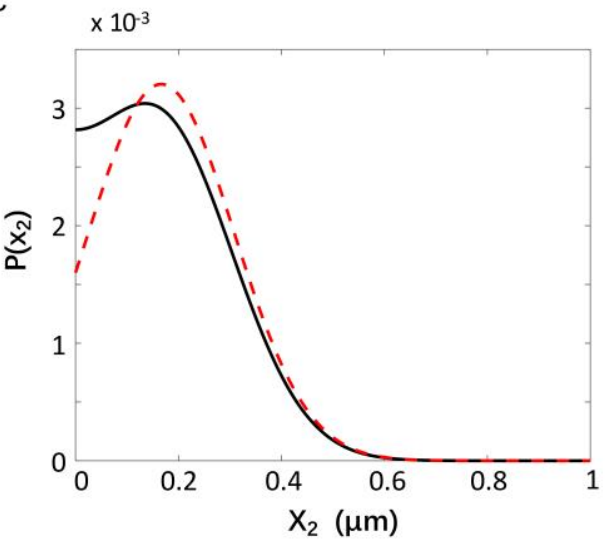

Figure S1. The probability distribution of one-dimensional restricted diffusion when $L$ equals to $1 \mu \mathrm{m}$ (a) and the sectional view of it when $x_{1}=1 / 2 \mu \mathrm{m}$ (b), or $x_{1}=$ $1 / 6 \mu \mathrm{m}$ (c). In (b) and (c), the black line is the actual probability distribution of restricted diffusion, the red dotted line is the probability distribution of Brownian diffusion with the same diffusion coefficient. 

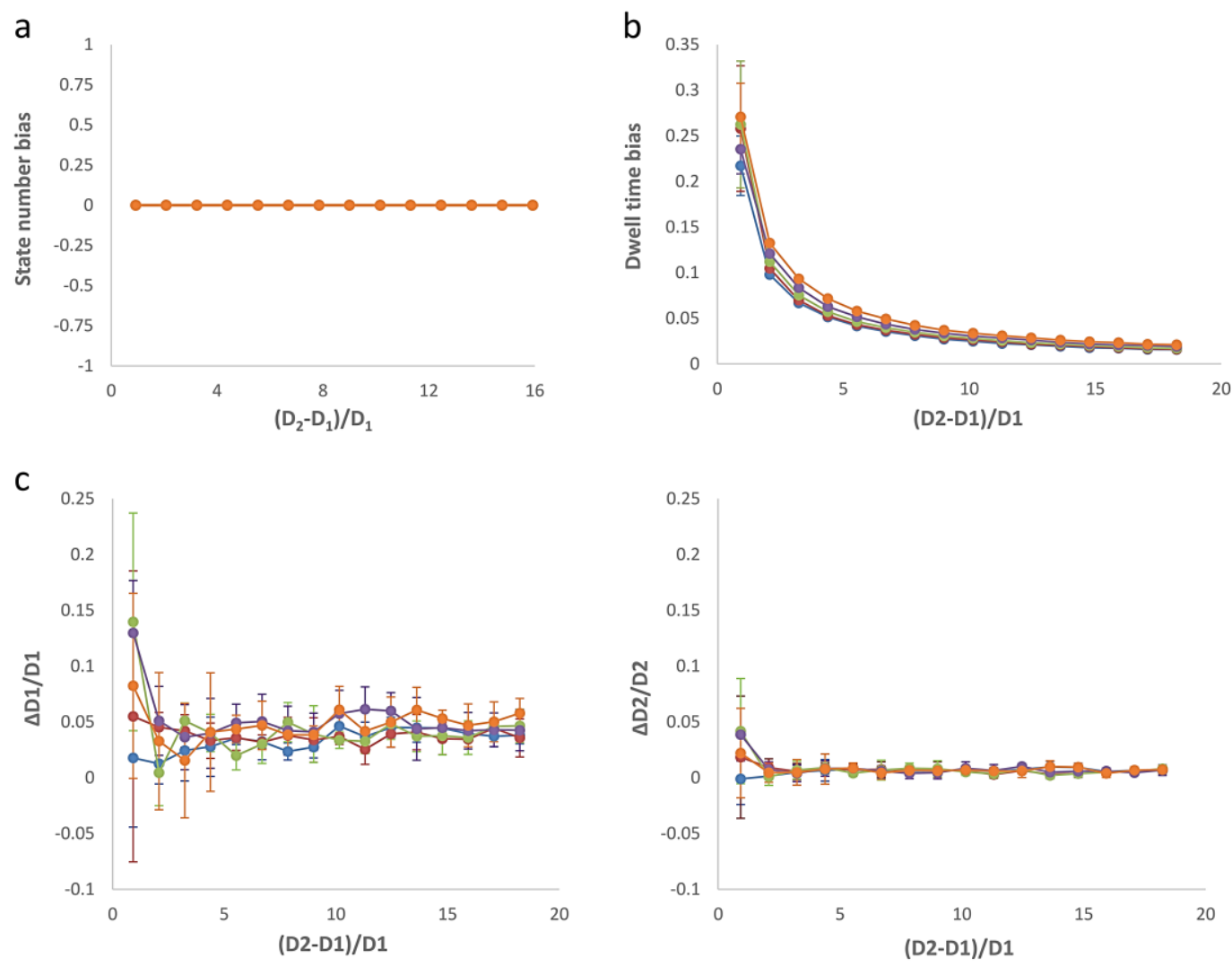

Figure S2. When the localization uncertainty $\sqrt{\left\langle(\Delta x)^{2}\right\rangle}$ varied from 10, 20, 30, 40 to $50 \mathrm{~nm}$ (blue, red, green, purple, orange respectively), changes of parameter biases and variances under different diffusion coefficient separation with bootstrapping number 11. (a) Biases and standard deviations of the state number. (b) Biases and standard deviations of the state dwell time. (c) Biases and standard deviations of the diffusion coefficients. 

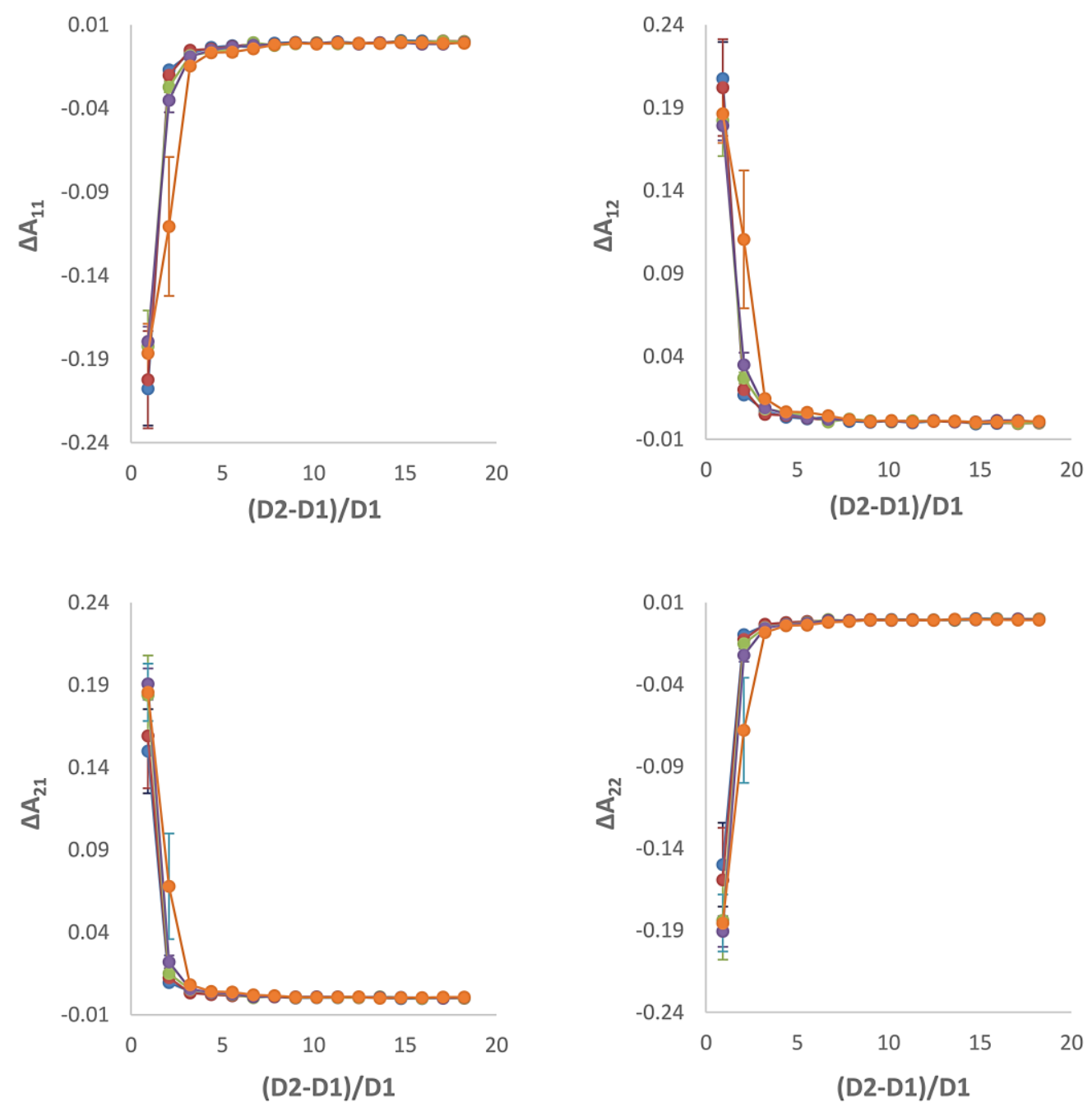

Figure S3. When the localization uncertainty $\sqrt{\left\langle(\Delta x)^{2}\right\rangle}$ varied from 10 to $50 \mathrm{~nm}$ (blue, red, green, purple, orange respectively), changes of biases and variances of the transition probabilities under different diffusion coefficients separation with bootstrapping number 11. 
a

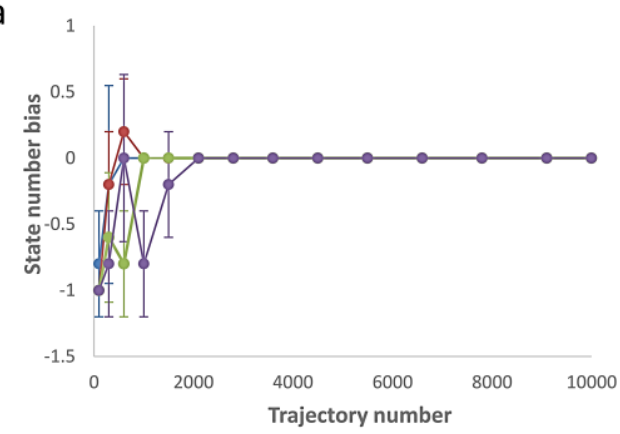

b

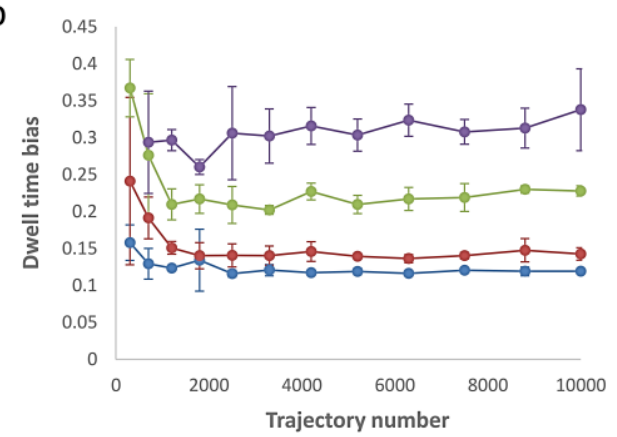

C
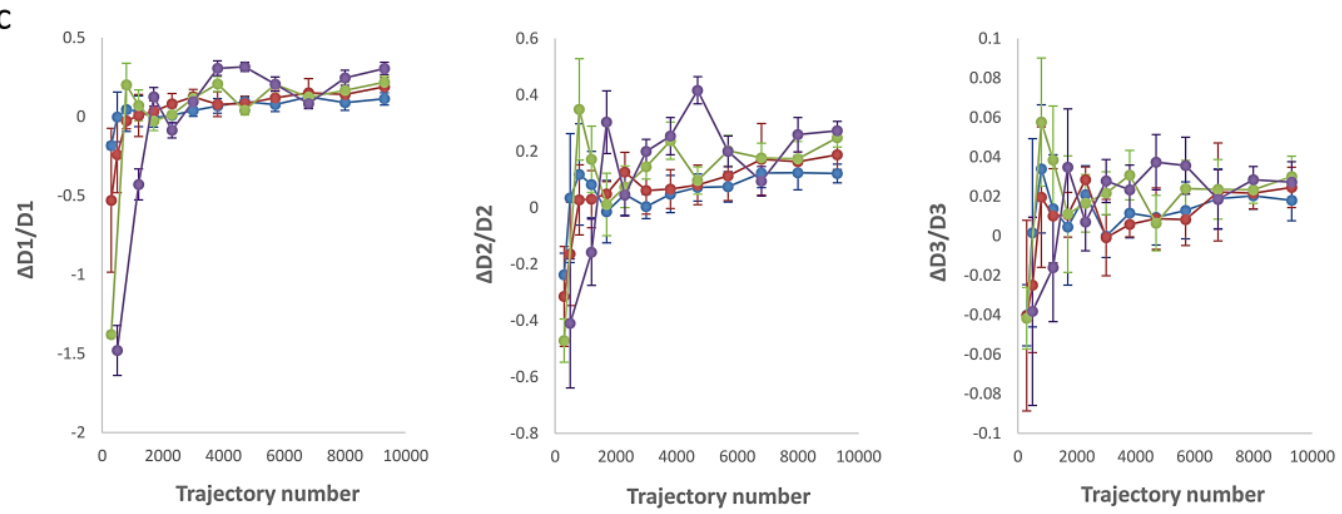

Figure S4. With the localization uncertainty $\sqrt{\left\langle(\Delta x)^{2}\right\rangle}$ varied from $10 \mathrm{~nm}, 20 \mathrm{~nm}, 30 \mathrm{~nm}$ to $40 \mathrm{~nm}$ (blue, red, green, purple respectively), changes of parameter biases and variances under varied trajectory number with bootstrapping number 11. (a) Biases and standard deviations of the state number. (b) Biases and standard deviations of the state dwell time. (c) Biases and standard deviations of the diffusion coefficients. 

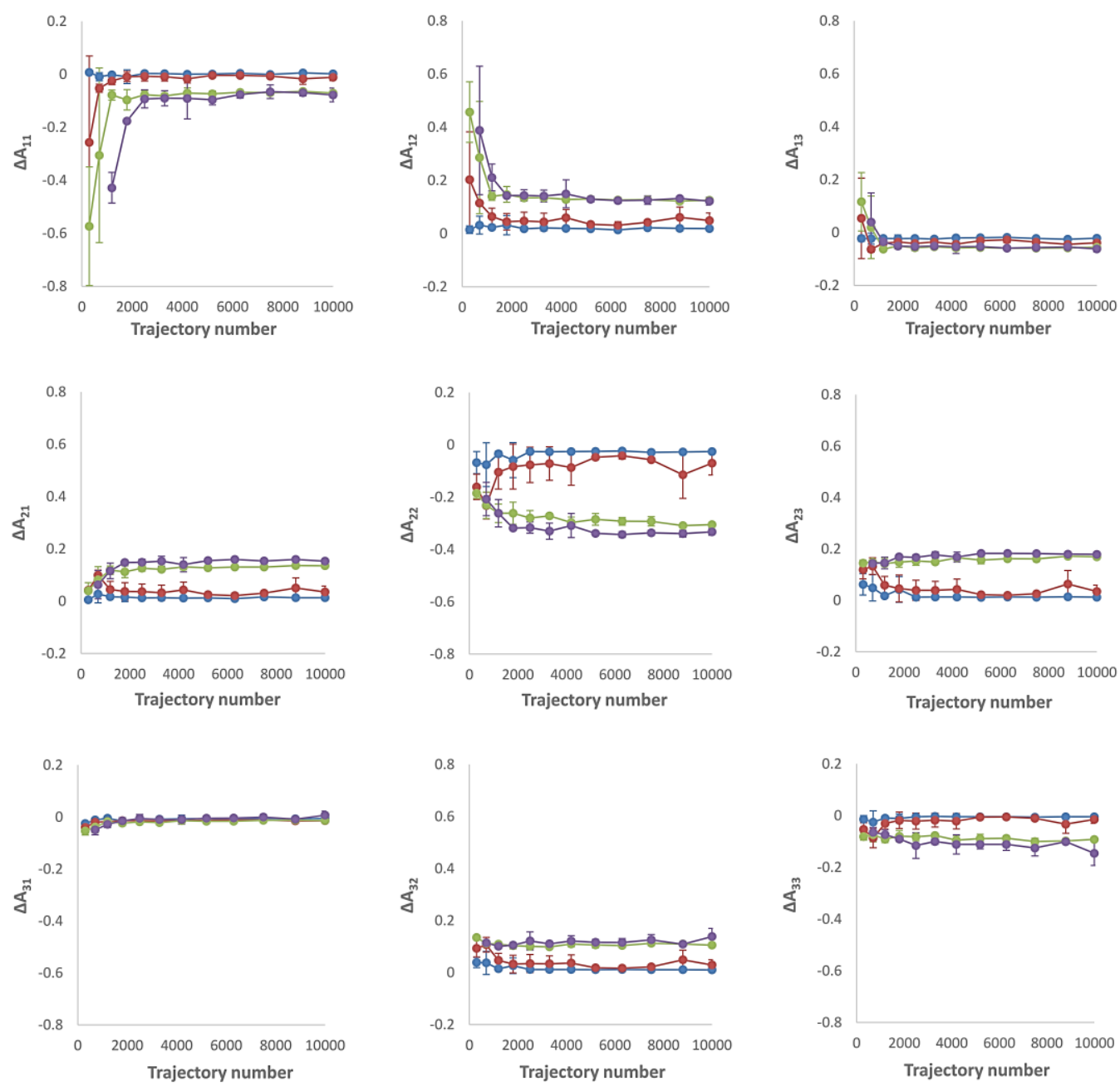

Figure S5. With the localization uncertainty $\sqrt{\left\langle(\Delta x)^{2}\right\rangle}$ varied from $10 \mathrm{~nm}, 20 \mathrm{~nm}, 30 \mathrm{~nm}$ to $40 \mathrm{~nm}$ (blue, red, green, purple respectively), changes of biases and variances of the transition probabilities under varied trajectory number with bootstrapping number 11 . 

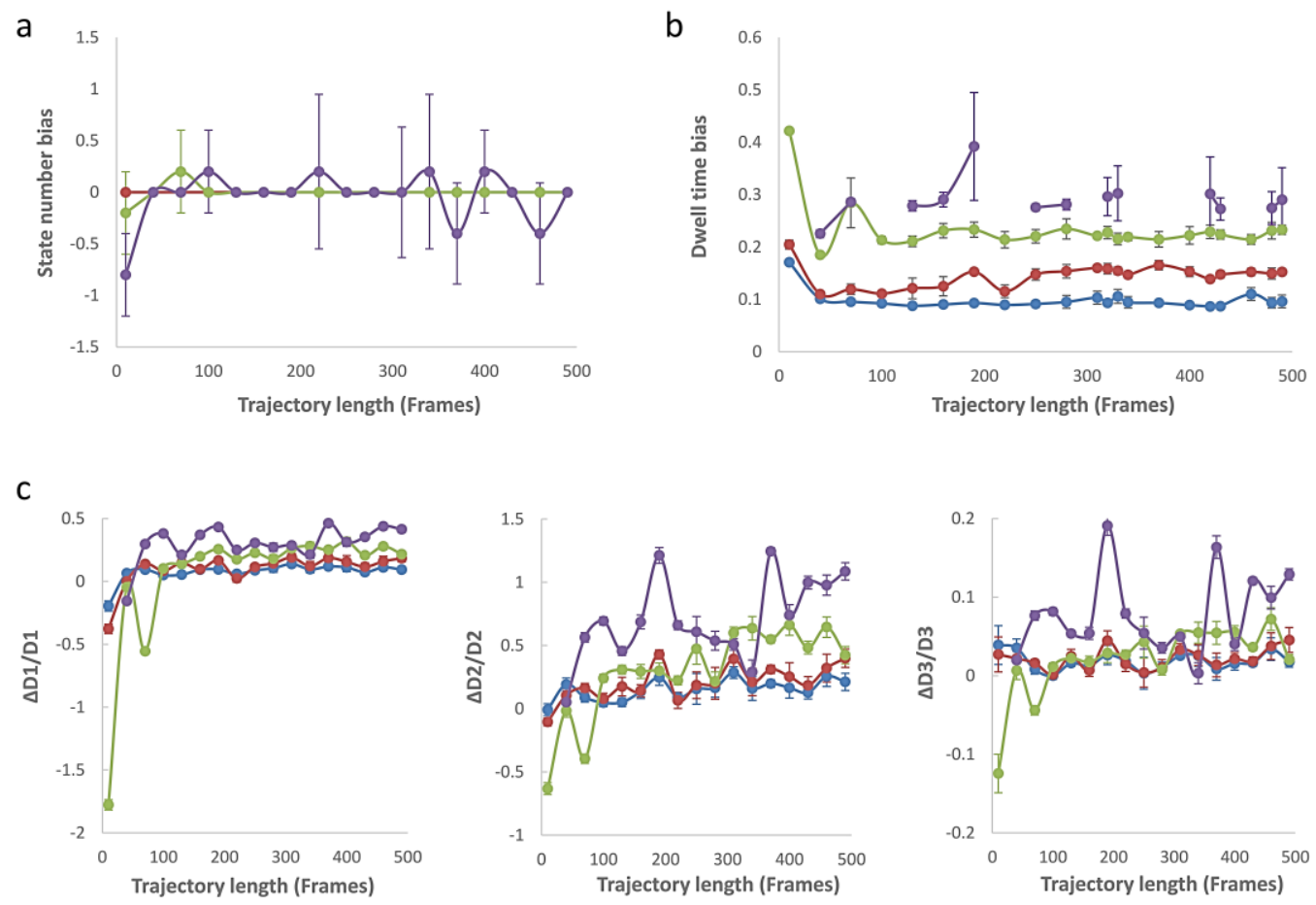

Figure S6. With the localization uncertainty $\sqrt{\left\langle(\Delta x)^{2}\right\rangle}$ varied from $10 \mathrm{~nm}, 20 \mathrm{~nm}, 30 \mathrm{~nm}$ to $40 \mathrm{~nm}$ (blue, red, green, purple respectively), change of parameter biases and variances under varied single trajectory length with bootstrapping number 11. (a) Biases and standard deviations of the state number. (b) Biases and standard deviations of the state dwell time (Note: when the uncertainty is large such as $40 \mathrm{~nm}$, the state number could be sometimes falsely predicted, thus the dwell time bias could not be calculated in those cases, resulting in the disconnected purple line). (c) Biases and standard deviations of the diffusion coefficients. 

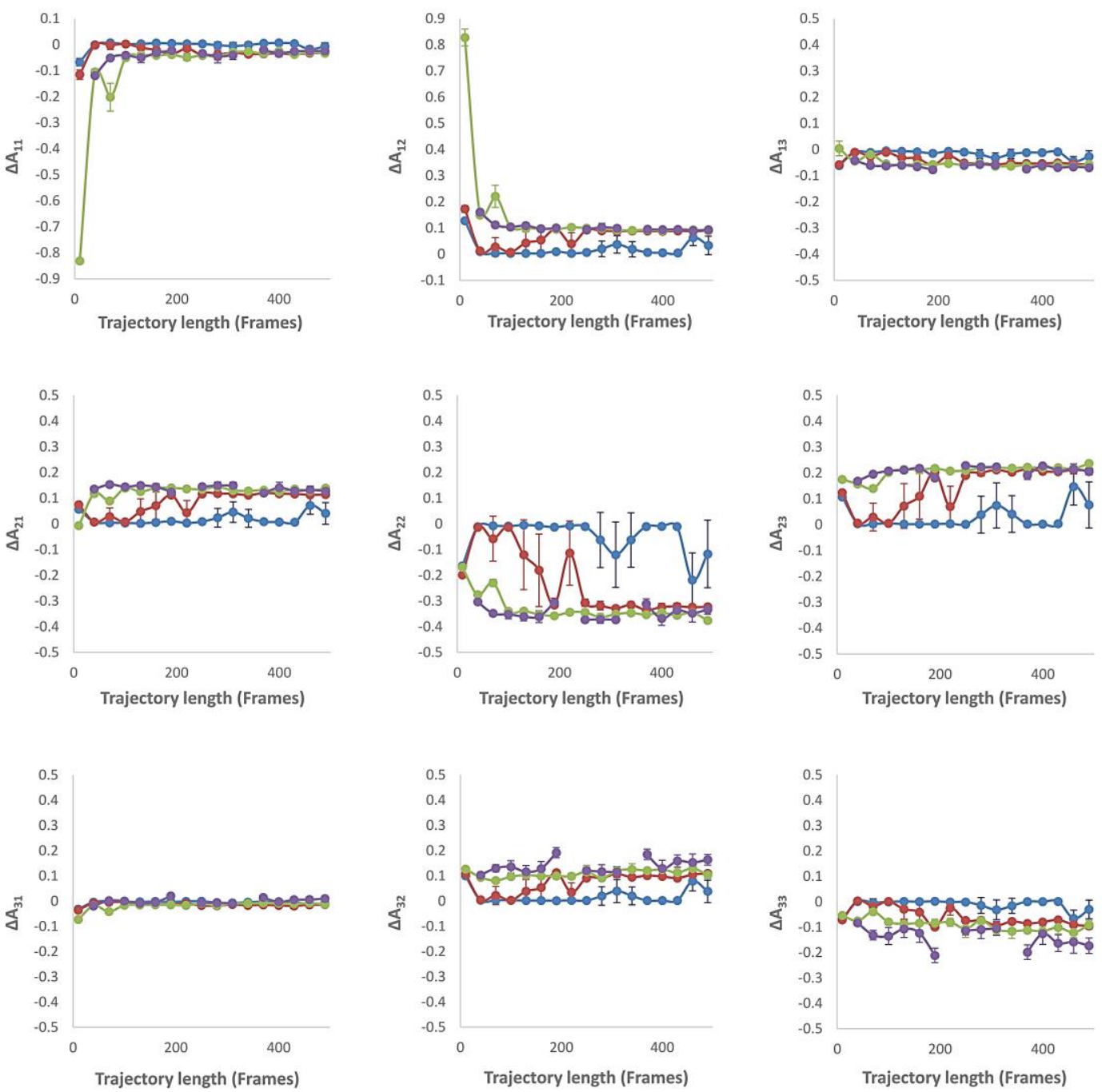

Figure S7. With the localization uncertainty $\sqrt{\left\langle(\Delta x)^{2}\right\rangle}$ varied from $10 \mathrm{~nm}, 20 \mathrm{~nm}, 30 \mathrm{~nm}$ to $40 \mathrm{~nm}$ (blue, red, green, purple respectively), changes of biases and variances of the transition probabilities under varied single trajectory length with bootstrapping number 11 . 


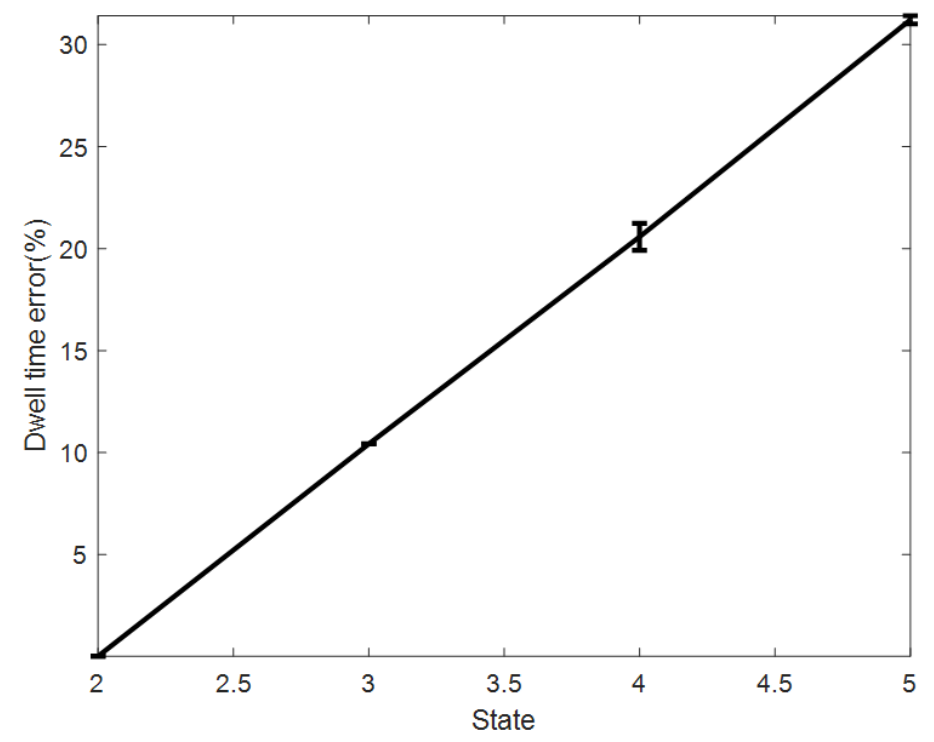

Figure S8. Changes of the state dwell time bias and standard variances under different state number with bootstrapping number 5 .

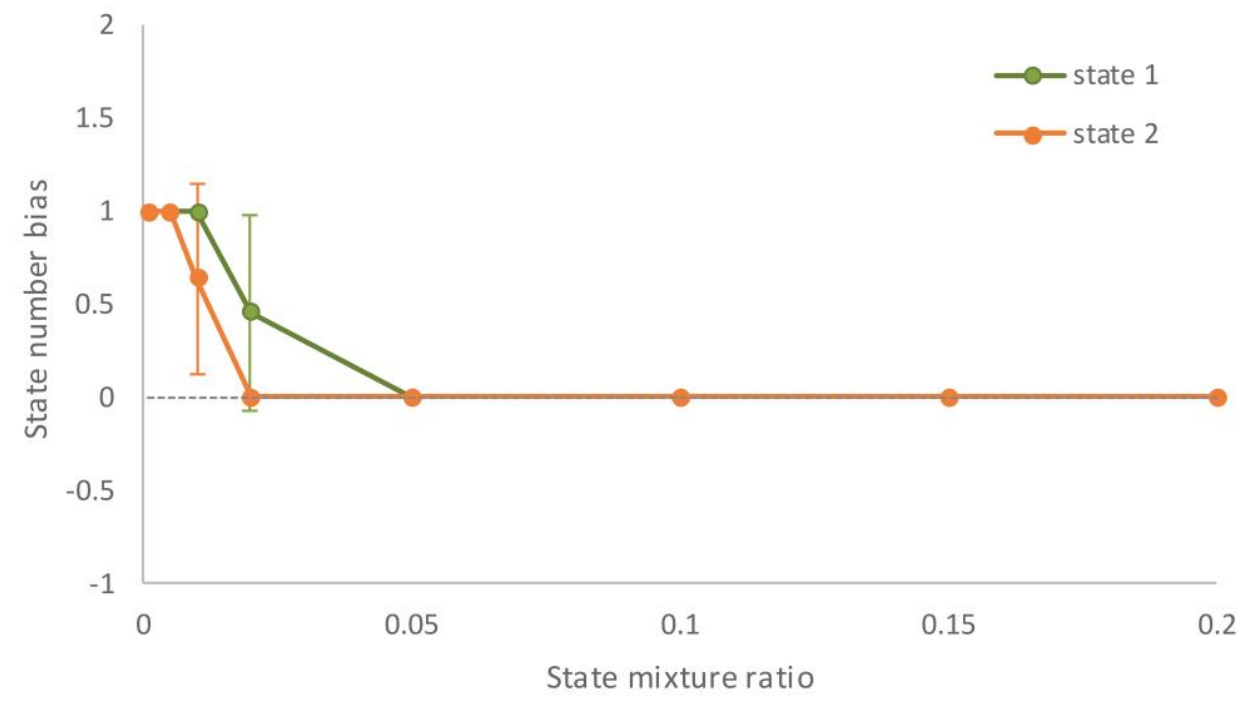

Figure S9. Changes of the state number bias and standard variances under different state mixture ratio with bootstrapping number 11 . 

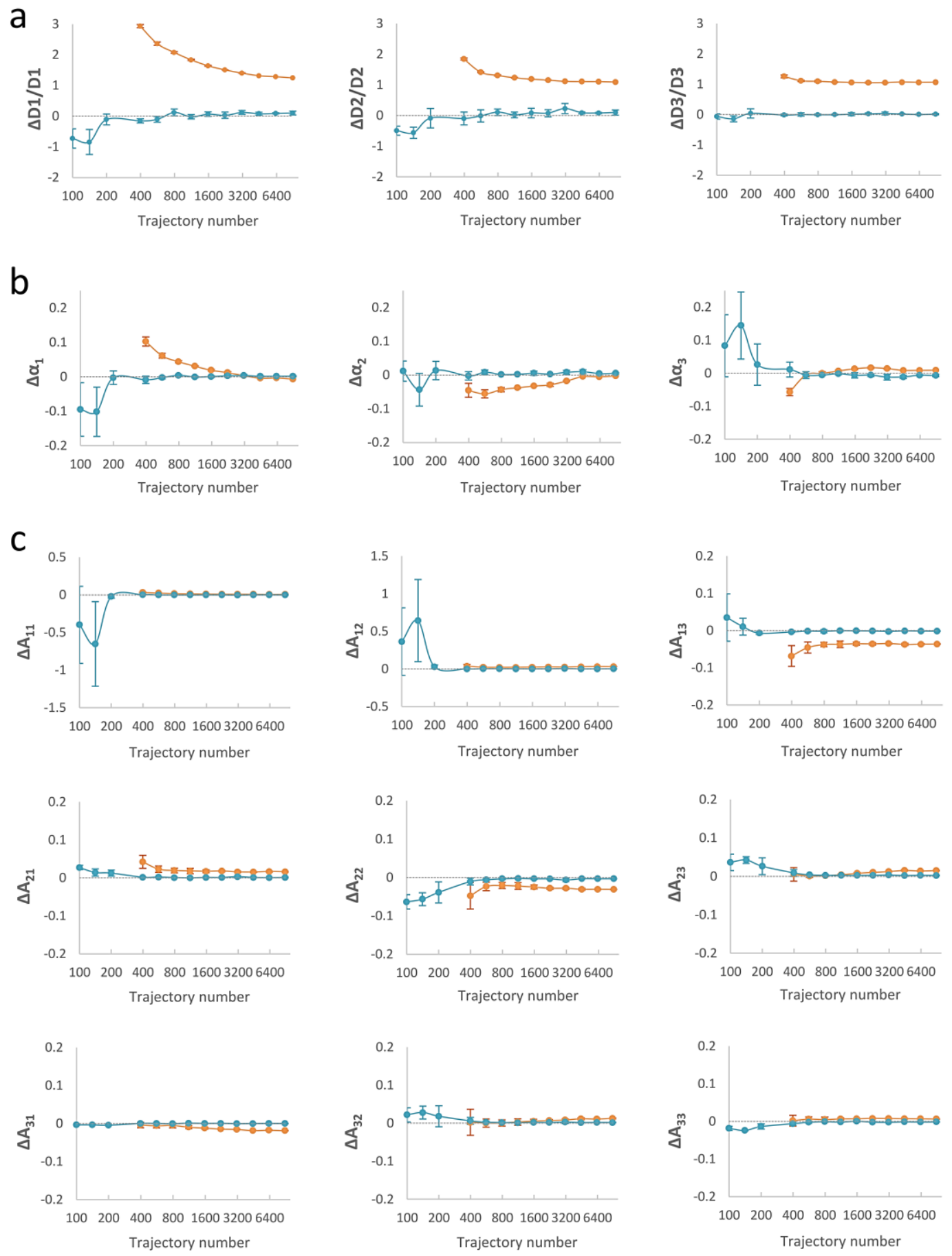

Figure S10. Comparison of parameter biases of vbSPT (orange lines) and RmHMM (blue lines) under varied trajectory number for the synthesized series of 3-state diffusion trajectories (Table S8). The error bars are the standard deviations of three sets of data. (a) Biases of the diffusion coefficients. (b) Biases of the state mixture ratio. (c) Biases of the transition probabilities. The results of vbSPT were not shown when trajectory number was less than 400 , because it falsely identified two diffusion states. 

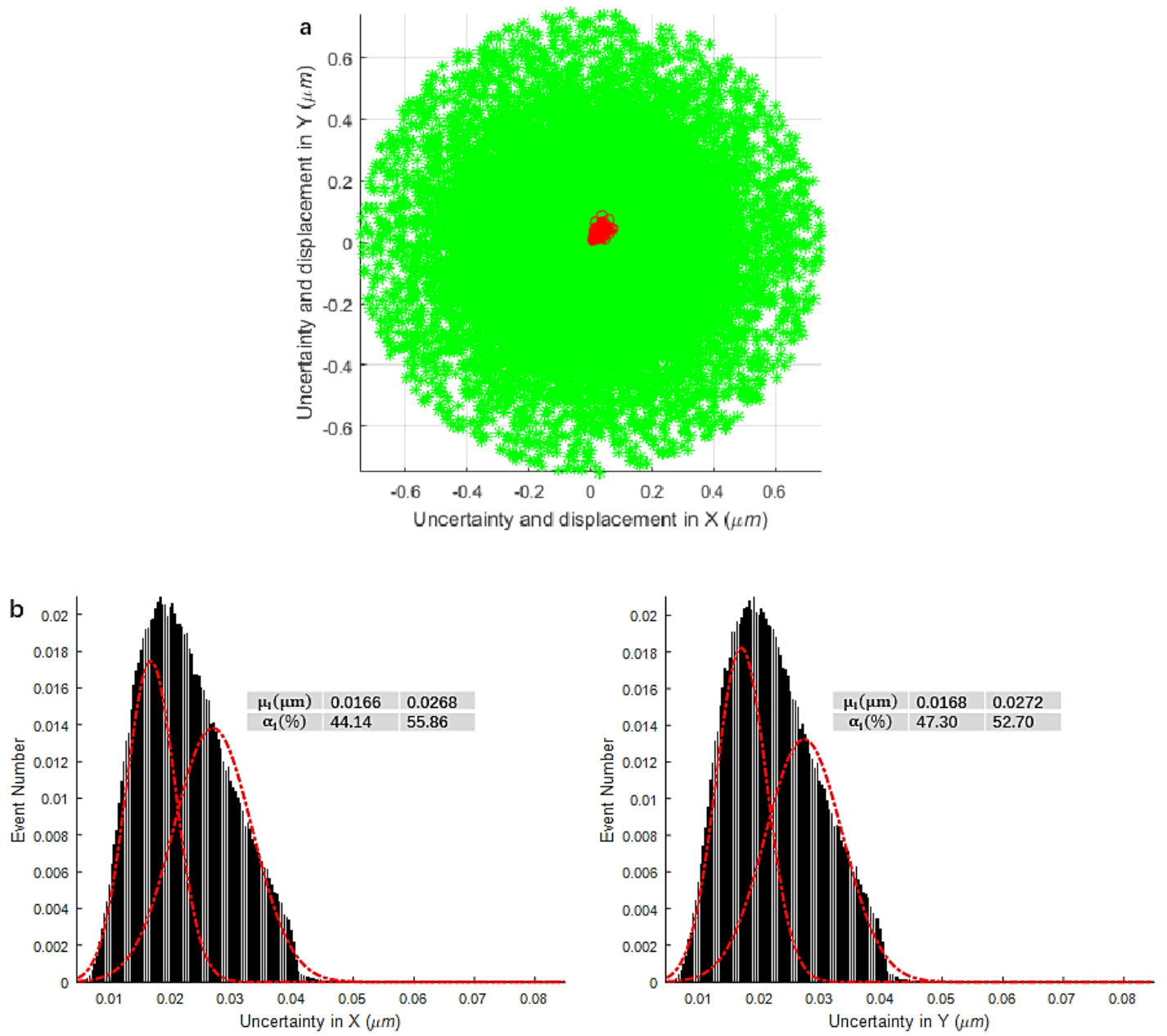

Figure S11. Diffusion displacement and localization uncertainty distribution calculated from EGFR data. (a) The comparison between diffusion displacement (green stars) and localization uncertainty (red circles). (b) The localization uncertainty distribution (black bars) in X (left) and Y (right) dimensions and the fitted Gaussian mixture models (red dashed curves).

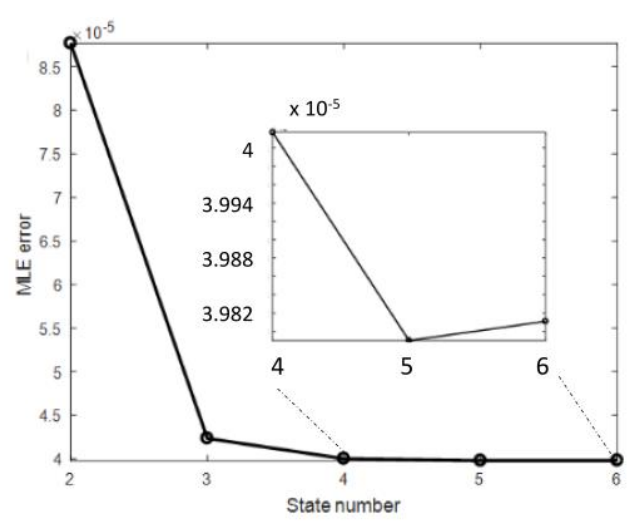

b

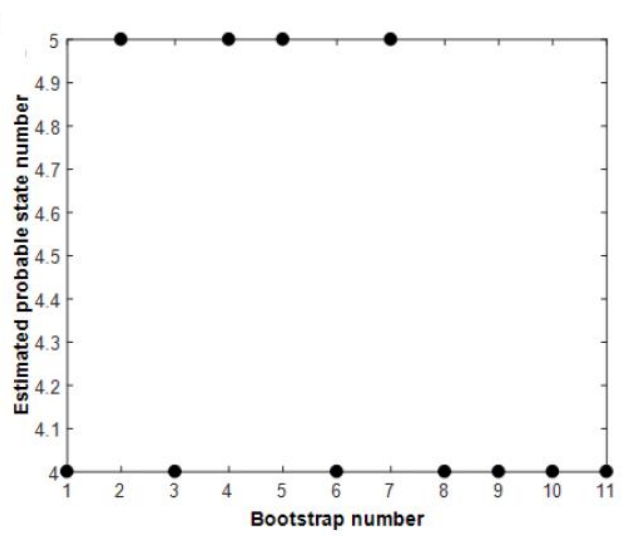

Figure S12. The estimation of state number K from the MLE (a) Changes of MLE error with increasing state number. The insert figure is the magnified view of state number from 4 to 6 . (b) Bootstrapping process of calculating the state number. 


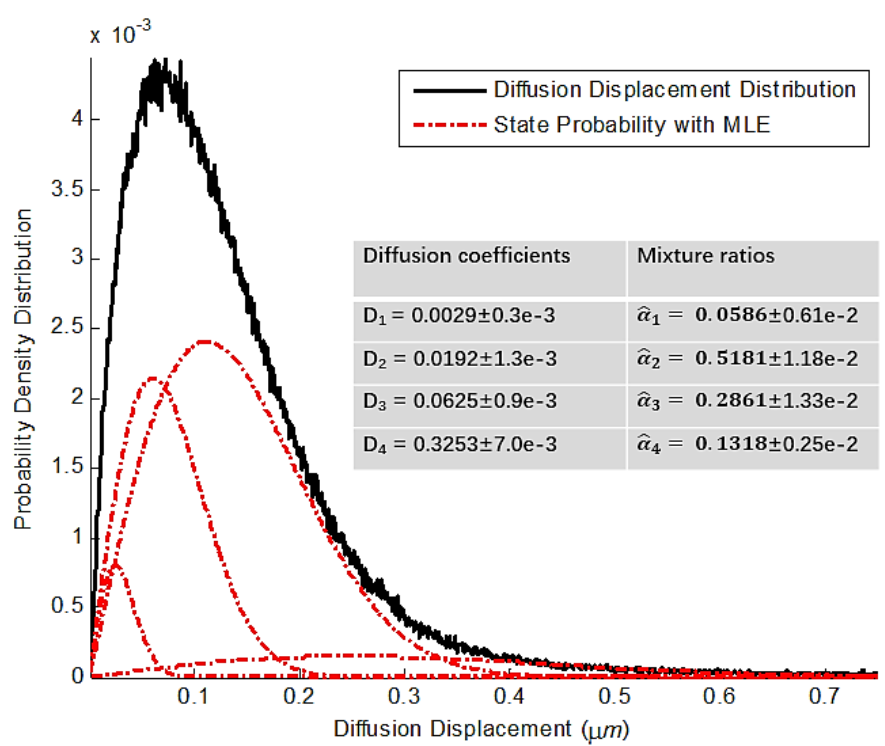

Figure S13. The probability density distribution and its estimated 4-state Rayleigh mixture distribution of experimental data from EGFR imaging in resting and stimulated HeLa cells. The inset table are the estimated parameters with MLE.
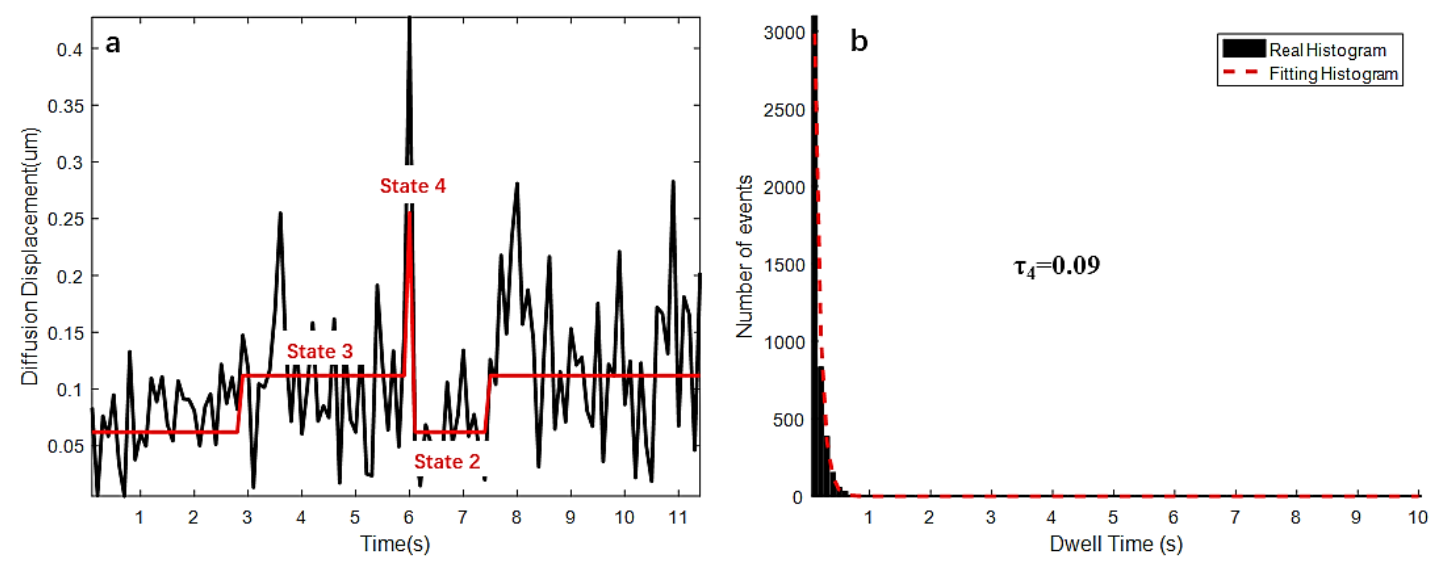

Figure S14. A representative trajectory of single EGFR molecule with unusual transition between the state 3 and state 4 (a) and the exponential decay of the dwell time of the $4^{\text {th }}$ state (b). 


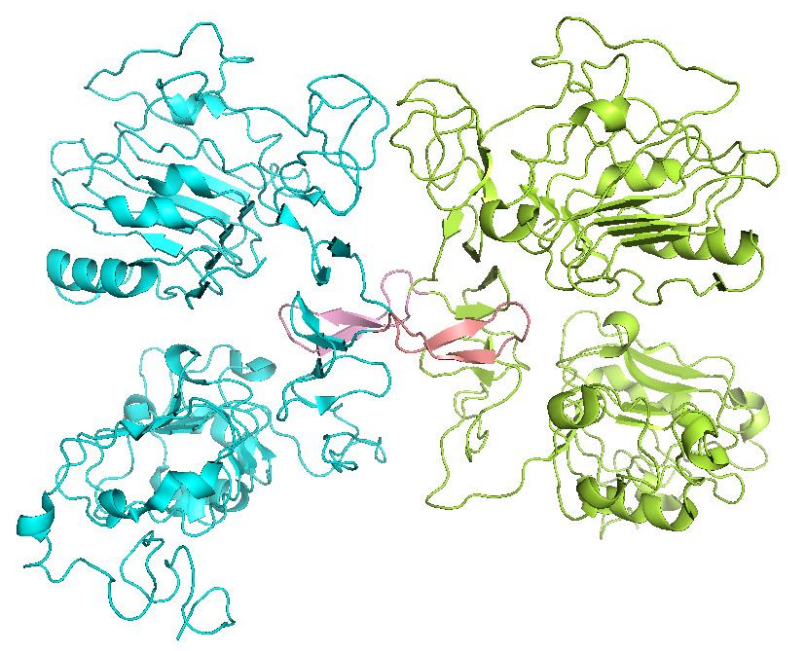

Figure S15. Ribbon diagram of the dimeric EGFR extracellular domain structure. The dimerization arms (residues 242-259) of two receptors are shown in pink and red, respectively. (PDB accession no. 3LTF)

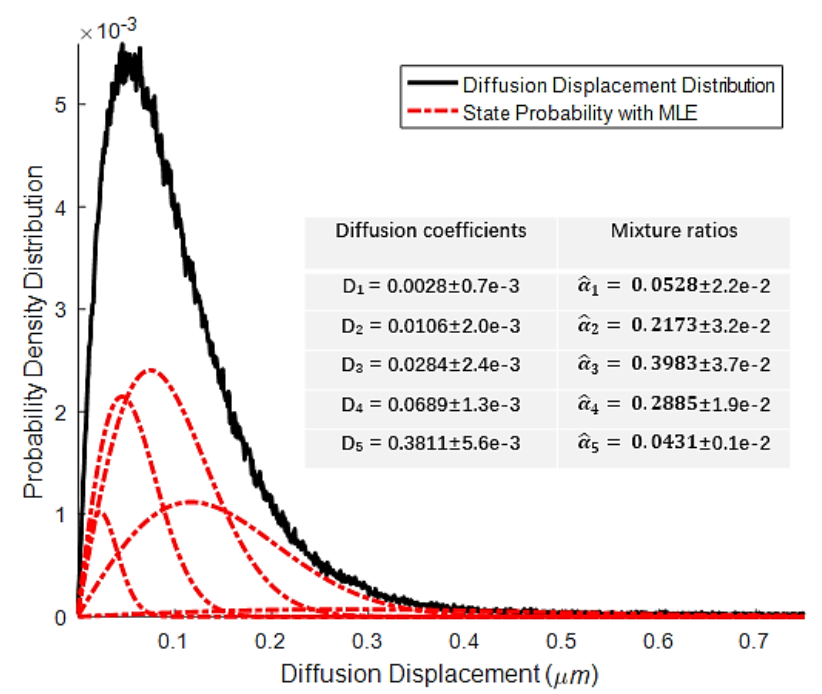

Figure S16. The probability density distribution and its estimated 5-state Rayleigh mixture distribution of experimental data from TRRII imaging in resting and stimulated HeLa cells. The inset table displayed the estimated parameters with MLE. 

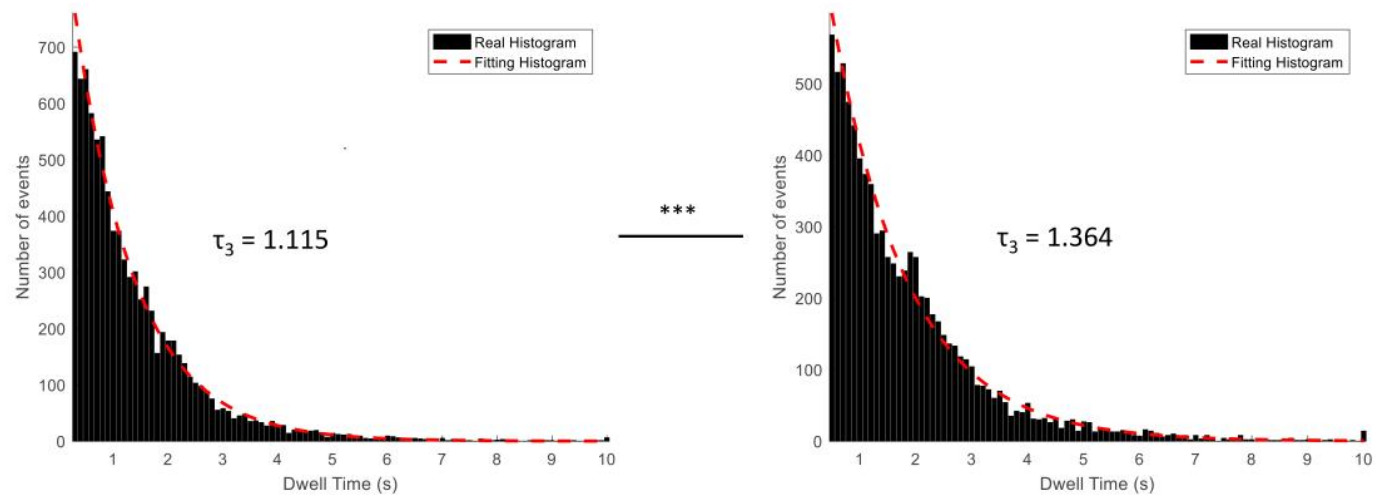

Figure S17. The dwell time of the dimeric T $\beta$ RII before (left panel) and after (right panel) ligand stimulation. The histograms were fitted with a single exponential function. The dotted lines are the fitting curves. The $\tau$ values obtained from cells with ligand stimulations were compared to those of the resting cells using Mann-Whiteney $\mathrm{U}$ test. ${ }^{* * *}, \mathrm{P}<0.001$. 
Table S1. The extracted parameters in four diffusion states from EGFR data in resting and stimulated HeLa cells.

\begin{tabular}{|c|c|c|c|}
\hline $\begin{array}{l}\text { Diffusion coefficients } \\
\left(\mu \mathrm{m}^{2} / \mathrm{s}\right)\end{array}$ & Cell states & Mixture ratios (\%) & State transition probability \\
\hline \multirow{2}{*}{$D_{1}=0.0029 \pm 0.3 \mathrm{e}-3$} & Resting & $\alpha_{1}=0.19 \pm 0.2$ & $\begin{array}{l}\mathrm{A}_{11}=0.2523 \pm 0.1322 \\
\mathrm{~A}_{12}=0.4044 \pm 0.0927 \\
\mathrm{~A}_{13}=0.2753 \pm 0.1769 \\
\mathrm{~A}_{14}=0.0681 \pm 0.0471\end{array}$ \\
\hline & Stimulated & $\alpha_{1}=0.92 \pm 0.79$ & $\begin{array}{l}\mathrm{A}_{11}=0.3721 \pm 0.1423 \\
\mathrm{~A}_{12}=0.4215 \pm 0.0208 \\
\mathrm{~A}_{13}=0.1753 \pm 0.1291 \\
\mathrm{~A}_{14}=0.0311 \pm 0.0284\end{array}$ \\
\hline \multirow{2}{*}{$D_{2}=0.0192 \pm 1.3 \mathrm{e}-3$} & Resting & $\alpha_{2}=9.74 \pm 3.48$ & $\begin{array}{l}\mathrm{A}_{21}=0.0448 \pm 0.0055 \\
\mathrm{~A}_{22}=0.6906 \pm 0.0942 \\
\mathrm{~A}_{23}=0.2216 \pm 0.0677 \\
\mathrm{~A}_{24}=0.0430 \pm 0.0299\end{array}$ \\
\hline & Stimulated & $\alpha_{2}=25.48 \pm 5.85$ & $\begin{array}{l}\mathrm{A}_{21}=0.0531 \pm 0.0090 \\
\mathrm{~A}_{22}=0.7713 \pm 0.0738 \\
\mathrm{~A}_{23}=0.1537 \pm 0.0565 \\
\mathrm{~A}_{24}=0.0219 \pm 0.0154\end{array}$ \\
\hline \multirow{2}{*}{$D_{3}=0.0625 \pm 0.9 \mathrm{e}-3$} & Resting & $\alpha_{3}=83.81 \pm 3.67$ & $\begin{array}{l}\mathrm{A}_{31}=0.0105 \pm 0.0083 \\
\mathrm{~A}_{32}=0.0634 \pm 0.0157 \\
\mathrm{~A}_{33}=0.8160 \pm 0.0310 \\
\mathrm{~A}_{34}=0.1101 \pm 0.0333\end{array}$ \\
\hline & Stimulated & $\alpha_{3}=68.46 \pm 6.65$ & $\begin{array}{l}\mathrm{A}_{31}=0.0118 \pm 0.0095 \\
\mathrm{~A}_{32}=0.0772 \pm 0.0175 \\
\mathrm{~A}_{33}=0.8107 \pm 0.0316 \\
\mathrm{~A}_{34}=0.1003 \pm 0.0383\end{array}$ \\
\hline \multirow{2}{*}{$D_{4}=0.3273 \pm 1.3 \mathrm{e}-2$} & Resting & $\alpha_{4}=6.26 \pm 1.11$ & $\begin{array}{l}\mathrm{A}_{41}=0.0111 \pm 0.0082 \\
\mathrm{~A}_{42}=0.0776 \pm 0.0345 \\
\mathrm{~A}_{43}=0.5616 \pm 0.0834 \\
\mathrm{~A}_{44}=0.3497 \pm 0.0605\end{array}$ \\
\hline & Stimulated & $\alpha_{4}=5.14 \pm 1.01$ & $\begin{array}{l}\mathrm{A}_{41}=0.0109 \pm 0.0083 \\
\mathrm{~A}_{42}=0.0780 \pm 0.0293 \\
\mathrm{~A}_{43}=0.5439 \pm 0.0836 \\
\mathrm{~A}_{44}=0.3671 \pm 0.0795\end{array}$ \\
\hline
\end{tabular}


Table S2. The extracted parameters in four diffusion states from DelD data in resting and stimulated HeLa cells.

\begin{tabular}{|c|c|c|c|}
\hline $\begin{array}{l}\text { Diffusion coefficients } \\
\left(\mu \mathrm{m}^{2} / \mathrm{s}\right)\end{array}$ & Cell states & Mixture ratios (\%) & State transition probability \\
\hline \multirow{2}{*}{$D_{1}=0.0034 \pm 0.4 \mathrm{e}-3$} & Resting & $\alpha_{1}=0.53 \pm 0.82$ & $\begin{array}{l}\mathrm{A}_{11}=0.2511 \pm 0.2140 \\
\mathrm{~A}_{12}=0.3850 \pm 0.0249 \\
\mathrm{~A}_{13}=0.3049 \pm 0.1598 \\
\mathrm{~A}_{14}=0.0590 \pm 0.0337\end{array}$ \\
\hline & Stimulated & $\alpha_{1}=0.45 \pm 0.58$ & $\begin{array}{l}\mathrm{A}_{11}=0.2578 \pm 0.1870 \\
\mathrm{~A}_{12}=0.4164 \pm 0.0450 \\
\mathrm{~A}_{13}=0.2818 \pm 0.1333 \\
\mathrm{~A}_{14}=0.0440 \pm 0.0201\end{array}$ \\
\hline \multirow{2}{*}{$D_{2}=0.0213 \pm 2.5 \mathrm{e}-3$} & Resting & $\alpha_{2}=22.34 \pm 2.23$ & $\begin{array}{l}\mathrm{A}_{21}=0.0519 \pm 0.0010 \\
\mathrm{~A}_{22}=0.7282 \pm 0.0257 \\
\mathrm{~A}_{23}=0.1937 \pm 00232 \\
\mathrm{~A}_{24}=0.0263 \pm 0.0041\end{array}$ \\
\hline & Stimulated & $\alpha_{2}=26.72 \pm 5.15$ & $\begin{array}{l}\mathrm{A}_{21}=0.0533 \pm 0.0052 \\
\mathrm{~A}_{22}=0.7489 \pm 0.0145 \\
\mathrm{~A}_{23}=0.1779 \pm 0.0169 \\
\mathrm{~A}_{24}=0.0199 \pm 0.0010\end{array}$ \\
\hline \multirow{2}{*}{$D_{3}=0.0691 \pm 5 \mathrm{e}-3$} & Resting & $\alpha_{3}=72.67 \pm 1.68$ & $\begin{array}{l}\mathrm{A}_{31}=0.018 \pm 0.0093 \\
\mathrm{~A}_{32}=0.0874 \pm 0.0138 \\
\mathrm{~A}_{33}=0.8143 \pm 0.0266 \\
\mathrm{~A}_{34}=0.0800 \pm 0.0154\end{array}$ \\
\hline & Stimulated & $\alpha_{3}=69.29 \pm 5.01$ & $\begin{array}{l}\mathrm{A}_{31}=0.0190 \pm 0.0103 \\
\mathrm{~A}_{32}=0.0911 \pm 0.0199 \\
\mathrm{~A}_{33}=0.8194 \pm 0.0280 \\
\mathrm{~A}_{34}=0.0706 \pm 0.0119\end{array}$ \\
\hline \multirow{2}{*}{$D_{4}=0.3167 \pm 2.2 \mathrm{e}-2$} & Resting & $\alpha_{4}=4.45 \pm 1.25$ & $\begin{array}{l}\mathrm{A}_{41}=0.0161 \pm 0.0081 \\
\mathrm{~A}_{42}=0.0872 \pm 0.0052 \\
\mathrm{~A}_{43}=0.5025 \pm 0.0487 \\
\mathrm{~A}_{44}=0.3941 \pm 0.0368\end{array}$ \\
\hline & Stimulated & $\alpha_{4}=3.53 \pm 0.33$ & $\begin{array}{l}\mathrm{A}_{41}=0.0170 \pm 0.0098 \\
\mathrm{~A}_{42}=0.0883 \pm 0.0164 \\
\mathrm{~A}_{43}=0.5127 \pm 0.0305 \\
\mathrm{~A}_{44}=0.3819 \pm 0.0233\end{array}$ \\
\hline
\end{tabular}


Table S3. Model parameters for figure 1.

\begin{tabular}{|c|l|}
\hline Parameters & Values \\
\hline$K$ & 3 \\
\hline$D_{1}$ & 0.0057 \\
\hline$D_{2}$ & 0.0260 \\
\hline$D_{3}$ & 0.0790 \\
\hline$\pi_{1}$ & 0.23 \\
\hline$\pi_{2}$ & 0.34 \\
\hline$\pi_{3}$ & 0.43 \\
\hline$A_{11}$ & 0.839 \\
\hline$A_{12}$ & 0.011 \\
\hline$A_{13}$ & 0.150 \\
\hline$A_{21}$ & 0.014 \\
\hline$A_{22}$ & 0.976 \\
\hline$A_{23}$ & 0.010 \\
\hline$A_{31}$ & 0.075 \\
\hline$A_{32}$ & 0.003 \\
\hline$A_{33}$ & 0.922 \\
\hline$N$ & 10000 \\
\hline$L$ & 50 \\
\hline$\sqrt{\left\langle(\Delta x)^{2}\right\rangle}$ & $0 \mathrm{~nm}$ \\
\hline & \\
\hline
\end{tabular}

Table S4. Model parameters for checking the dependence of recovery accuracy on the diffusion coefficients separation.

\begin{tabular}{|c|l|}
\hline Parameters & Values \\
\hline$K$ & 2 \\
\hline$D_{1}$ & 0.026 \\
\hline$D_{2}$ & $0.050-0.500$ \\
\hline$\pi_{1}$ & 0.43 \\
\hline$\pi_{2}$ & 0.57 \\
\hline$A_{11}$ & 0.939 \\
\hline$A_{12}$ & 0.061 \\
\hline$A_{21}$ & 0.024 \\
\hline$A_{22}$ & 0.976 \\
\hline$N$ & 3000 \\
\hline$L$ & 50 \\
\hline$\sqrt{\left\langle(\Delta x)^{2}\right\rangle}$ & $20 \mathrm{~nm}$ \\
\hline
\end{tabular}


Table S5. Model parameters for checking the dependence of recovery accuracy on the trajectory number.

\begin{tabular}{|c|l|}
\hline Parameters & Values \\
\hline$K$ & 3 \\
\hline$D_{1}$ & 0.0057 \\
\hline$D_{2}$ & 0.0260 \\
\hline$D_{3}$ & 0.0790 \\
\hline$\pi_{1}$ & 0.23 \\
\hline$\pi_{2}$ & 0.34 \\
\hline$\pi_{3}$ & 0.43 \\
\hline$A_{11}$ & 0.839 \\
\hline$A_{12}$ & 0.011 \\
\hline$A_{13}$ & 0.150 \\
\hline$A_{21}$ & 0.014 \\
\hline$A_{22}$ & 0.976 \\
\hline$A_{23}$ & 0.010 \\
\hline$A_{31}$ & 0.075 \\
\hline$A_{32}$ & 0.003 \\
\hline$A_{33}$ & 0.922 \\
\hline$N$ & $100-10000$ \\
\hline$L$ & 20 \\
\hline$\sqrt{\left\langle(\Delta x)^{2}\right\rangle}$ & $20 \mathrm{~nm}$ \\
\hline
\end{tabular}


Table S6. Model parameters for checking the recovery accuracy at different trajectory length.

\begin{tabular}{|c|l|}
\hline Parameters & Values \\
\hline$K$ & 3 \\
\hline$D_{1}$ & 0.0057 \\
\hline$D_{2}$ & 0.0260 \\
\hline$D_{3}$ & 0.0790 \\
\hline$\pi_{1}$ & 0.23 \\
\hline$\pi_{2}$ & 0.34 \\
\hline$\pi_{3}$ & 0.43 \\
\hline$A_{11}$ & 0.839 \\
\hline$A_{12}$ & 0.011 \\
\hline$A_{13}$ & 0.150 \\
\hline$A_{21}$ & 0.014 \\
\hline$A_{22}$ & 0.976 \\
\hline$A_{23}$ & 0.010 \\
\hline$A_{31}$ & 0.075 \\
\hline$A_{32}$ & 0.003 \\
\hline$A_{33}$ & 0.922 \\
\hline$N$ & 1000 \\
\hline$L$ & $10-500$ \\
\hline$\sqrt{\left\langle(\Delta x)^{2}\right\rangle}$ & $20 \mathrm{~nm}$ \\
\hline & \\
\hline
\end{tabular}

Table S7. Model parameters for checking the recovery accuracy at different state mixture ratio.

\begin{tabular}{|c|l|}
\hline Parameters & Values \\
\hline$K$ & 2 \\
\hline$D_{1}$ & 0.0260 \\
\hline$D_{2}$ & 0.0790 \\
\hline$\pi_{1}$ & $0.001-0.20,0.80-0.999$ \\
\hline$\pi_{2}$ & $0.001-0.20,0.80-0.999$ \\
\hline$A_{11}$ & 1.0 \\
\hline$A_{12}$ & 0.0 \\
\hline$A_{21}$ & 0.0 \\
\hline$A_{22}$ & 1.0 \\
\hline$N$ & 2000 \\
\hline$L$ & 50 \\
\hline$\sqrt{\left\langle(\Delta x)^{2}\right\rangle}$ & $10 \mathrm{~nm}$ \\
\hline & \\
\hline
\end{tabular}


Table S8. Model parameters for comparing the performance of RmHMM and vbSPT.

\begin{tabular}{|c|l|}
\hline Parameters & Values \\
\hline$K$ & 3 \\
\hline$D_{1}$ & 0.0057 \\
\hline$D_{2}$ & 0.0260 \\
\hline$D_{3}$ & 0.0790 \\
\hline$\pi_{1}$ & 0.23 \\
\hline$\pi_{2}$ & 0.34 \\
\hline$\pi_{3}$ & 0.43 \\
\hline$A_{11}$ & 0.839 \\
\hline$A_{12}$ & 0.011 \\
\hline$A_{13}$ & 0.150 \\
\hline$A_{21}$ & 0.014 \\
\hline$A_{22}$ & 0.976 \\
\hline$A_{23}$ & 0.010 \\
\hline$A_{31}$ & 0.075 \\
\hline$A_{32}$ & 0.003 \\
\hline$A_{33}$ & 0.922 \\
\hline$N$ & $100-10000$ \\
\hline$L$ & $2-40$ \\
\hline$L$ mean & 20 \\
\hline$\sqrt{\left\langle(\Delta x)^{2}\right\rangle}$ & $20 \mathrm{~nm}$ \\
\hline & \\
\hline
\end{tabular}

Table S9. The parameters for training RmHMM with the mixing data of EGFR in Hela cells

\begin{tabular}{|c|c|c|c|}
\hline Experiment No. & 1 & 2 & 3 \\
\hline$N$ & 8902 & 4174 & 5140 \\
\hline$L$ & $\geq 20$ & $\geq 20$ & $\geq 20$ \\
\hline$\sqrt{\left\langle(\Delta x)^{2}\right\rangle}$ & $22.3 \mathrm{~nm}$ & $20.5 \mathrm{~nm}$ & $23.2 \mathrm{~nm}$ \\
\hline
\end{tabular}

Table S10. The parameters for training RmHMM with the mixing data of DelD in Hela cells

\begin{tabular}{|c|c|c|c|}
\hline Experiment No. & 1 & 2 & 3 \\
\hline$N$ & 5033 & 14396 & 8658 \\
\hline$L$ & $\geq 20$ & $\geq 20$ & $\geq 20$ \\
\hline$\sqrt{\left\langle(\Delta x)^{2}\right\rangle}$ & $19.4 \mathrm{~nm}$ & $22.6 \mathrm{~nm}$ & $20.3 \mathrm{~nm}$ \\
\hline
\end{tabular}

Table S11. The parameters for training RmHMM with the mixing data of T $\beta R I I$ in Hela cells

\begin{tabular}{|c|c|c|c|}
\hline Experiment No. & 1 & 2 & 3 \\
\hline$N$ & 7589 & 8145 & 11479 \\
\hline$L$ & $\geq 20$ & $\geq 20$ & $\geq 20$ \\
\hline$\sqrt{\left\langle(\Delta x)^{2}\right\rangle}$ & $24.6 \mathrm{~nm}$ & $21.1 \mathrm{~nm}$ & $19.8 \mathrm{~nm}$ \\
\hline
\end{tabular}


Table S12. The extracted parameters in five diffusion states from TßRII data in resting and stimulated HeLa cells.

\begin{tabular}{|c|c|c|c|}
\hline $\begin{array}{l}\text { Diffusion coefficients } \\
\left(\mu \mathrm{m}^{2} / \mathrm{s}\right)\end{array}$ & Cell states & Mixture ratios (\%) & State transition probability \\
\hline \multirow{2}{*}{$D_{1}=0.0028 \pm 0.7 \mathrm{e}-3$} & Resting & $\alpha_{1}=0.92 \pm 0.35$ & $\begin{array}{l}\mathrm{A}_{11}=0.6492 \pm 0.0460 \\
\mathrm{~A}_{12}=0.2615 \pm 0.0253 \\
\mathrm{~A}_{13}=0.0657 \pm 0.0180 \\
\mathrm{~A}_{14}=0.0068 \pm 0.0012 \\
\mathrm{~A}_{15}=0.0168 \pm 0.0037\end{array}$ \\
\hline & Stimulated & $\alpha_{1}=1.31 \pm 1.35$ & $\begin{array}{l}\mathrm{A}_{11}=0.7052 \pm 0.0362 \\
\mathrm{~A}_{12}=0.2489 \pm 0.0373 \\
\mathrm{~A}_{13}=0.0389 \pm 0.0055 \\
\mathrm{~A}_{14}=0.0030 \pm 0.0010 \\
\mathrm{~A}_{15}=0.0039 \pm 0.0023\end{array}$ \\
\hline \multirow{2}{*}{$D_{2}=0.0106 \pm 2.0 \mathrm{e}-3$} & Resting & $\alpha_{2}=10.07 \pm 0.75$ & $\begin{array}{l}\mathrm{A}_{21}=0.0427 \pm 0.0032 \\
\mathrm{~A}_{22}=0.7900 \pm 0.0167 \\
\mathrm{~A}_{23}=0.1448 \pm 0.0165 \\
\mathrm{~A}_{24}=0.0161 \pm 0.0012 \\
\mathrm{~A}_{25}=0.0066 \pm 0.0015\end{array}$ \\
\hline & Stimulated & $\alpha_{2}=14.99 \pm 0.92$ & $\begin{array}{l}\mathrm{A}_{21}=0.0400 \pm 0.0111 \\
\mathrm{~A}_{22}=0.8161 \pm 0.0134 \\
\mathrm{~A}_{23}=0.1300 \pm 0.0078 \\
\mathrm{~A}_{24}=0.0113 \pm 0.0015 \\
\mathrm{~A}_{25}=0.0026 \pm 0.0015\end{array}$ \\
\hline \multirow{2}{*}{$D_{3}=0.0284 \pm 2.4 \mathrm{e}-3$} & Resting & $\alpha_{3}=53.53 \pm 4.23$ & $\begin{array}{l}\mathrm{A}_{31}=0.0039 \pm 0.0002 \\
\mathrm{~A}_{32}=0.0475 \pm 0.0036 \\
\mathrm{~A}_{33}=0.7993 \pm 0.0131 \\
\mathrm{~A}_{34}=0.1189 \pm 0.0130 \\
\mathrm{~A}_{35}=0.0304 \pm 0.0028\end{array}$ \\
\hline & Stimulated & $\alpha_{3}=60.73 \pm 1.25$ & $\begin{array}{l}\mathrm{A}_{31}=0.0026 \pm 0.0015 \\
\mathrm{~A}_{32}=0.0524 \pm 0.0035 \\
\mathrm{~A}_{33}=0.8312 \pm 0.0099 \\
\mathrm{~A}_{34}=0.0999 \pm 0.0043 \\
\mathrm{~A}_{35}=0.0138 \pm 0.0056\end{array}$ \\
\hline \multirow{2}{*}{$D_{4}=0.0689 \pm 1.3 \mathrm{e}-3$} & Resting & $\alpha_{4}=31.97 \pm 3.99$ & $\begin{array}{l}\mathrm{A}_{41}=0.0006 \pm 0.0002 \\
\mathrm{~A}_{42}=0.0071 \pm 0.0021 \\
\mathrm{~A}_{43}=0.1502 \pm 0.0343 \\
\mathrm{~A}_{44}=0.7650 \pm 0.0363 \\
\mathrm{~A}_{45}=0.0771 \pm 0.0062\end{array}$ \\
\hline & Stimulated & $\alpha_{4}=21.4 \pm 2.49$ & $\begin{array}{l}\mathrm{A}_{41}=0.0003 \pm 0.0002 \\
\mathrm{~A}_{42}=0.0087 \pm 0.0011 \\
\mathrm{~A}_{43}=0.1786 \pm 0.0025 \\
\mathrm{~A}_{44}=0.7652 \pm 0.0159 \\
\mathrm{~A}_{45}=0.0472 \pm 0.0139\end{array}$ \\
\hline
\end{tabular}




\begin{tabular}{|l|l|l|l|}
\hline & & & $\mathrm{A}_{51}=0.0082 \pm 0.0012$ \\
& \multirow{5}{*}{$D_{5}=0.3811 \pm 5.6 \mathrm{e}-3$} & & $\alpha_{52}=0.0205 \pm 0.0066$ \\
& & & $\mathrm{~A}_{53}=0.2551 \pm 0.0582$ \\
$\mathrm{~A}_{54}=0.4052 \pm 0.0577$ \\
\cline { 3 - 4 } & & & $\mathrm{A}_{55}=0.3110 \pm 0.0106$ \\
\hline \multirow{5}{*}{ Stimulated } & $\alpha_{5}=1.57 \pm 0.74$ & $\mathrm{~A}_{51}=0.0048 \pm 0.0048$ \\
& & & $\mathrm{~A}_{52}=0.0236 \pm 0.0050$ \\
& & & $\mathrm{~A}_{53}=0.2824 \pm 0.0155$ \\
& & $\mathrm{~A}_{54}=0.4193 \pm 0.0385$ \\
& & $\mathrm{~A}_{55}=0.2700 \pm 0.0252$ \\
\hline
\end{tabular}




\section{Supporting References}

(1) Kusumi, A.; Sako, Y.; Yamamoto, M. Confined lateral diffusion of membrane receptors as studied by single particle tracking (nanovid microscopy) effects of calcium-induced differentiation in cultured epithelial cells. Biophysical Journal. 1993, 65, 2021-40.

(2) Bickel T. A note on confined diffusion. Physica A, 2007, 377, 24-32.

(3) Persson, F.; Linden, M.; Unoson, C.; Elf, J. Extracting intracellular diffusive states and transition rates from single-molecule tracking data. Nat Methods. 2013, 10, 265-269.

(4) Thompson, R. E.; Larson, D. R.; Webb, W. W. Precise nanometer localization analysis for individual fluorescent probes. Biophysical Journal. 2002, 82, 2775-2783.

(5) Zhang, W.; Jiang, Y.; Wang, Q.; Ma, X.; Xiao, Z.; Zuo, W.; Fang, X.; Chen, Y. G. Single-molecule imaging reveals transforming growth factor- $\beta$-induced type II receptor dimerization. Proc Natl Acad Sci U.S.A. 2009, 106, 15679-83.

(6) Mattoon, D.; Klein, P.; Lemmon, M. A.; Lax, I.; Schlessinger, J. The tethered configuration of the egf receptor extracellular domain exerts only a limited control of receptor function. Proc Natl Acad Sci U.S.A. 2004, 101, 923-928.

(7) Jaqaman, K.; Loerke, D.; Mettlen, M.; Kuwata, H.; Grinstein, S.; Schmid, S. L.; Danuser, G. Robust single-particle tracking in live-cell time-lapse sequences. Nat Methods. 2008, 5, 695-702. 